\title{
Theoretical Aspects of Light Meson Spectroscopy*
}

\author{
T. Barnes \\ Computational and Theoretical Physics Group, Oak Ridge National Laboratory \\ Oak Ridge, TN 37831-6373, USA \\ and \\ Department of Physics and Astronomy, University of Tennessee \\ Knoxville, TN 37996-1200, USA
}

\begin{abstract}
In this pedagogical review we discuss the theoretical understanding of light hadron spectroscopy in terms of QCD and the quark model. We begin with a summary of the known and surmised properties of QCD and confinement. Following this we review the nonrelativistic quark potential model for $q \bar{q}$ mesons and discuss the quarkonium spectrum and methods for identifying $q \bar{q}$ states. Finally we review theoretical expectations for non- $q \bar{q}$ states (glueballs, hybrids and multiquark systems) and the status of experimental candidates for these states.
\end{abstract}

\footnotetext{
*This is a summary of lectures given at the Nato Advanced Study Institute on Hadron Spectroscopy and the Confinement Problem, 27 June - 8 July 1995, at Queen Mary and Westfield College, London and the University of Swansea. 


\section{INTRODUCTION}

\section{A. The QCD and QED Lagrangians}

Quantum chromodynamics (QCD) describes the strong interaction as a quantum field theory of interacting spin-1/2 Dirac fermions (the quarks) and eight vector gauge fields (the gluons). (For a review of early QCD references see Marciano and Pagels [1].) There are six different types "flavors" of quark, which have different masses, electric charges and weak interactions but identical strong interactions. For each of these flavors of quarks there is an internal label called "color", $i=1,2,3$, and the three colors of quarks are coupled to the $a=1 . .8$ colors of gluons in a manner described by the QCD Lagrangian. The QCD Lagrangian density for the gluons and a single quark flavor is

$$
\mathcal{L}_{Q C D}=\bar{\psi}_{i}\left(\delta_{i j}\left(i \not \partial-m_{q}\right)-g \frac{\lambda_{i j}^{a}}{2} \not A^{a}\right) \psi_{j}-\frac{1}{4} F_{\mu \nu}^{a} F_{\mu \nu}^{a}
$$

where the gluon field strength tensor $F_{\mu \nu}^{a}$ is defined in terms of the gluon field by

$$
F_{\mu \nu}^{a}=\partial_{\nu} A_{\mu}^{a}-\partial_{\mu} A_{\nu}^{a}+g f^{a b c} A_{\mu}^{b} A_{\nu}^{c}
$$

The $\left\{\lambda_{i j}^{a}\right\}$ are the $3 \times 3$ Gell-Mann SU(3) matrices which couple a quark, antiquark and gluon together in a "color-singlet" combination that is invariant under $\mathrm{SU}(3)$ gauge transformations. (These are mathematically analogous to the Pauli matrices in the isospin-conserving pion-nucleon interaction $\bar{\Psi} \vec{\tau} \Psi \cdot \vec{\pi}$. This coupling implies that the quark-gluon interaction has an internal SU(3) gauge symmetry.)

The QCD Lagrangian is quite similar to the QED form

$$
\begin{gathered}
\mathcal{L}_{Q E D}=\bar{\psi}(i \not \partial-m-e \not A) \psi-\frac{1}{4} F_{\mu \nu} F_{\mu \nu} \\
F_{\mu \nu}=\partial_{\nu} A_{\mu}-\partial_{\mu} A_{\nu}
\end{gathered}
$$

except that in QED there is only a single "color" degree of freedom. These two Lagrangians are invariant under the gauge transformations 


$$
\begin{aligned}
& \psi \rightarrow e^{i e \Lambda(x)} \psi \quad \text { (QED) } \\
& A_{\mu} \rightarrow A_{\mu}-\partial_{\mu} \Lambda(x) \quad(\mathrm{QED}) \\
& \psi_{i} \rightarrow e^{i g \frac{\lambda_{i_{2}}^{a}}{2} \Lambda^{a}(x)} \psi_{j} \quad(\mathrm{QCD}) \\
& A_{\mu}^{a} \rightarrow A_{\mu}^{a}-\partial_{\mu} \Lambda^{a}(x)+g f^{a b c} \Lambda^{b}(x) A_{\mu}^{c} \quad \text { (QCD). }
\end{aligned}
$$

The invariance of the Lagrangians under these gauge transformations is crucial, since without this symmetry, interacting theories involving vector bosons in three space dimensions are nonrenormalizable. With this gauge invariance only a finite number (three) of independent infinities arise in perturbative QED and QCD calculations of scattering amplitudes, so once these infinities are absorbed in the definitions of three quantities taken from experiment, the remaining physical quantities calculated are finite and can be compared with experiment.

In both theories the $\bar{\psi} \psi A$ interaction implies an elementary fermion-antifermion-vector vertex, the difference in QCD being the presence of the $\lambda_{i j}^{a}$ color matrix that gives the couplings of the different quark and gluon colors. The most important difference between QED and QCD is the presence in QCD of gluon self-couplings. Since the gluon field strength tensor $F_{\mu \nu}^{a}$ contains both linear and quadratic $A$ terms, its square in the QCD Lagrangian gives three-gluon and four-gluon interactions. These gluon self-interactions qualitatively change the nature of the spectrum of QCD relative to QED.

The lowest-order interaction between an electron-positron pair in QED is due to one photon exchange, which for slowly moving fermions gives the familiar $V(r)=-\alpha / r$ Coulomb potential. At next order in an expansion in $v^{2} / c^{2}$ this one photon exchange matrix element gives the spin-dependent terms of the Breit-Fermi Hamiltonian, spin-spin, spin-orbit and tensor. Since the strength of the electron-photon interaction at laboratory scales is quite weak, one photon exchange is a very good approximation. One may of course calculate the electron-positron interaction due to more complicated processes such as vacuum polarization loops and vertex corrections, which at the one-loop level in perturbation theory give $O\left(\alpha^{2}\right)$ 
contributions to the effective $e^{+} e^{-}$interaction. One finds that these one-loop corrections have a momentum dependence that make the electron-positron interaction appear slightly weaker with increasing distances than predicted by one photon exchange alone; this effect is known as screening, and gives logarithmic corrections to the $-\alpha / r$ potential.

In QCD there are additional one-loop corrections due to the gluon self-coupling, and these are large enough to change the sign of the screening effect relative to QED. This results in an effective quark "color charge" that increases with increasing separation between sources. This is evident in the increased $\alpha_{s}$ required in potential models to describe the spectroscopy of lighter quarks, which have wavefunctions with larger length scales; typically, fits to spectroscopy require $\alpha_{s}(u \bar{u}, d \bar{d}, s \bar{s}) \approx 0.6, \alpha_{s}(c \bar{c}) \approx 0.4$ and $\alpha_{s}(b \bar{b}) \approx 0.15$. This "antiscreening" is a perturbative indication of the presence of the remarkable effect known as confinement.

If we attempt to separate a quark-antiquark pair in a meson, we find that at large separations this antiscreening effect dominates the physics, and replaces the $1 / r$ term in the OGE color Coulomb interaction by what appears to be a linear potential,

$$
\lim _{r \rightarrow \infty} V_{q \bar{q}}(r)=b r
$$

where the constant $r$ is known as the "string tension", and is numerically found to be

$$
b \approx 0.18 \mathrm{GeV}^{2} \approx 0.9 \mathrm{GeV} / \mathrm{fm}
$$

The origins of confinement are poorly understood; it is widely believed to be a nonperturbative effect, and in lattice gauge theory simulations is seen to involve the formation of a "flux tube" of chaotic color fields between color charges. In potential models of mesons such as we will discuss the confining interaction is treated phenomenologically as a linear potential which has a Lorentz scalar coupling to quarks. (This is not obvious, and the earliest models assumed that the coupling was between timelike vector currents, as for the Coulomb interaction.) The combination of OGE color Coulomb and scalar confining interactions gives an effective potential for a slowly moving color-singlet $q \bar{q}$ pair of 


$$
V_{q \bar{q}}\left(r_{q \bar{q}}\right)=-\frac{4}{3} \frac{\alpha_{s}}{r}+b r .
$$

This potential is shown in Figure 1 with parameters typical of those found in fits to light $q \bar{q}$ systems, $\alpha_{s}=0.6$ and $b=0.18 \mathrm{GeV}^{2}$. The crossover from the perturbative OGE regime to separations at which confinement is dominant evidently takes place at $r_{q \bar{q}} \approx 0.4 \mathrm{fm}$.

An additional constant $V_{0}$ is usually added to this potential, and to fit light $q \bar{q}$ spectroscopy a large negative value of $V_{0} \approx-0.8 \mathrm{GeV}$ is required. This $V_{0}$ is largely needed to cancel the fictitious $2 m_{q}$ present when "consitituent" light quark masses of $\approx 330 \mathrm{MeV}$ are used in the Schrödinger equation, so we do not include a $V_{0}$ in the $V(r)$ of Figure 1.

\section{B. Color Singlets in QCD}

- Since the confining interaction operates to confine any colored objects, the physically observed hadrons must be color singlets. Since quarks transform as a fundamental representation 3 of the color $\mathrm{SU}(3)$ gauge group, and antiquarks as the conjugate representation $\overline{3}$, we may use group theory to determine the color representations of product states and search for the physically allowed color singlets. First, for two quarks,

$$
q \otimes q=3 \otimes 3=6 \oplus \overline{3}
$$

so "diquarks" cannot exist as separate hadrons. At the three quark level however we find

$$
q \otimes q \otimes q=10 \oplus 8 \oplus 8 \oplus 1,
$$

so three quark systems can exist as physical states; these are the conventional baryons, and the color-singlet wavefunction is the obvious contraction of three vectors to a singlet,

$$
|q q q(1)\rangle=\frac{1}{\sqrt{6}} \sum_{i, j, k=1}^{3}|i, j, k\rangle .
$$

Similarly any system of $q^{3 N}$ quarks has color singlet combinations, and can exist as physical states; the question is then the dynamical one of whether such multiquark systems are realized in nature as single multiquark clusters, or (as appears more likely) as loosely associated systems of $q^{3}$ color singlet baryons, such as the atomic nuclei. 
The same arguments applied to quark and antiquark systems tell us that $q \vec{q}$ can exist as a physical state,

$$
q \otimes \bar{q}=3 \otimes \overline{3}=8 \oplus 1
$$

which are the conventional $q \bar{q}$ quark model mesons. Again higher states are possible, and the first new color singlet which appears beyond $q \bar{q}$ in the quark-antiquark Hilbert space is in the combination $q^{2} \bar{q}^{2}$,

$$
q^{2} \otimes \bar{q}^{2} \subset 1
$$

and similarly for any combination of the form $\left(q^{3}\right)^{m}(q \bar{q})^{n}$. Although much effort in the early days of the quark model was expended on calculations of the properties of hypothetical multiquark hadrons such as $q^{2} \bar{q}^{2}$ "baryonia", $q^{6}$ "dibaryons" and so forth, it now appears that these may not exist as resonances (with rare exceptions) because they can be rearranged into separate $q \bar{q}$ mesons and $q^{3}$ baryons without interaction.

We can also look for color singlet basis states in combinations involving gluons, which obviously cannot be arranged into separate $q \bar{q}$ and $q^{3}$ systems. Since the gluon transforms as a color octet, the first physically allowed combination appears at the $g g$ level,

$$
g \otimes g=8 \otimes 8=27 \oplus 10 \oplus \overline{10} \oplus 8 \oplus 8 \oplus 1
$$

Since combining another gluon will repeat the $8 \otimes \delta \subset 1$ product, we can make physically allowed hadron basis states from any number of gluons greater than 1.

The final type of color-singlet combination involves quarks, antiquarks and gluons. Since $q \bar{q}$ contains a color octet, if we form the combination $q \bar{q} g$ we find a color singlet basis state,

$$
q \otimes \bar{q} \otimes g=3 \otimes \overline{3} \otimes 8=(8 \oplus 1) \otimes 8 \subset 1
$$

This $q \bar{q} g$ is the simplest possible color-singlet combination of quarks, antiquarks and gluons, and is known as a hybrid. We shall see that physical states made from this basis state and others can have quantum numbers forbidden to $q \bar{q}$ states, which makes the search for hybrids one of the most interesting topics in hadron spectroscopy today. 
Similarly one may form color singlet "hybrid baryon" basis states, since

$$
q \otimes q \otimes q \otimes g=3 \otimes 3 \otimes 3 \otimes \delta=(10 \oplus 8 \oplus 8 \oplus 1) \otimes 8 \subset 1,
$$

so hybrid baryon resonances are expected in addition to the conventional $q^{3}$ quark model baryons. Although the combinations $(q \bar{q})^{\ell}\left(q^{3}\right)^{m} g^{n}$ all contain color singlets for $\ell+m+n \geq 2$, they need not lead to additional hybrid resonances beyond $q \bar{q} g$ and $q^{3} g$ because of the fallapart effect discussed above for $q^{2} \bar{q}^{2}$.

\section{II. $Q \bar{Q}$ MESONS}

\section{A. $q \bar{q}$ States and Quantum Numbers}

We will begin our discussion of spectroscopy with a summary of the properties of the conventional quark model " $q \vec{q}$ " mesons, and we will specialize to mesons with identical quark and antiquark. The quarks are spin-1/2 Dirac fermions and have charges of $+2 e / 3$ (for $u, c, t$ quarks) and $-e / 3$ (for $d, s, b$ quarks). Our discussion will deal with the $(u, d)(\bar{u}, \bar{d}), d \bar{d}, s \bar{s}$ and $c \bar{c}$ systems. All these except $c \bar{c}$ are highly relativistic bound states and are rather similar physically; $c \bar{c}$ is only quasirelativistic and hence has a relatively simple level structure, which makes it a useful model system for our discussion of quark-antiquark forces. Since the $u$ and $d$ quarks are quite similar in mass, $\approx 5-10 \mathrm{MeV}$ and this is much smaller than the intrinsic mass scale of QCD itself, it is convenient to treat them as partners in an "isospin" doublet,

$$
[u, d]=[(1 / 2,1 / 2),(1 / 2,-1 / 2)]_{I, I_{z}},
$$

and an antiquark isodoublet, with a (-1) phase required to write the antiquark basis states (which transform according to the conjugate representation of $\mathrm{SU}(2)$ ) as members of the fundamental representation;

$$
[-\bar{d}, \bar{u}]=[(1 / 2,1 / 2),(1 / 2,-1 / 2)]_{I_{,} I_{z}} .
$$

Hadrons containing $s$ quarks have rather similar properties to $(u, d)$ systems, although the heavier strange quark mass of $\approx 150 \mathrm{MeV}$ means that the $s \bar{s}$ states are well displaced in 
mass from the $(u, d) q \bar{q}$ states, so with the exception of the $\eta-\eta^{\prime}$ system there appears to be little mixing between $s \bar{s}$ and light $q \bar{q}$ states.

When we form the physically allowed $q \bar{q}$ combinations, for light quarks we can make isospins $I=0,1$;

$$
q \otimes \bar{q}=2 \otimes 2=3 \oplus 1
$$

In $\mathrm{SU}(2)$ these representations are $2 I+1$ dimensional, and are $I=1$ and $I=0$ respectively. With the antiquark basis phases given above and conventional SU(2) Clebsch-Gordon coefficients these states are

$$
\left|I=1, I_{z}\right\rangle= \begin{cases}-|u \bar{d}\rangle & I_{z}=1 \\ \frac{1}{\sqrt{2}}(|u \bar{u}\rangle-|d \bar{d}\rangle), & I_{z}=0 \\ |d \bar{u}\rangle, & I_{z}=-1\end{cases}
$$

and

$$
\left|I=0, I_{z}=0\right\rangle=\frac{1}{\sqrt{2}}(|u \bar{u}\rangle+|d \bar{d}\rangle) .
$$

These isospin eigenstates are physically relevant because $q \bar{q}$ annihilation processes in QCD such as $u \bar{u} \leftrightarrow d \bar{d}$ are large relative to $m_{u}-m_{d}$ and so drive the physical states into the linear combinations $u \bar{u} \pm d \bar{d}$. In contrast, mixing with $s \bar{s}$ states is much less important, and where this mixing can be studied (as in the tensors) it is usually found to be quite small. The single well established exception is in the $\eta-\eta^{\prime}$ system, which is driven into approximately equal flavor mixtures of $s \bar{s}$ and nonstrange systems,

$$
|\eta\rangle \approx \frac{1}{\sqrt{2}}(|n \bar{n}\rangle-|s \bar{s}\rangle)
$$

and

$$
\left|\eta^{\prime}\right\rangle \approx \frac{1}{\sqrt{2}}(|n \bar{n}\rangle+|s \bar{s}\rangle)
$$

where $|n \bar{n}\rangle \equiv(|u \bar{u}\rangle+|d \bar{d}\rangle / \sqrt{2}$. These can be written in terms of a flavor mixing angle $\phi$ as

$$
|\eta\rangle=\cos (\phi)|n \bar{n}\rangle-\sin (\phi)|s \bar{s}\rangle
$$




$$
\left|\eta^{\prime}\right\rangle=\sin (\phi)|n \bar{n}\rangle+\cos (\phi)|s \bar{s}\rangle
$$

One often sees these states expressed as a linear combination of flavor SU(3) octet and singlet states, which was especially common in the early days of the quark model;

$$
\begin{aligned}
& |\eta\rangle=\cos (\theta)|8\rangle+\sin (\theta)|1\rangle, \\
& \left|\eta^{\prime}\right\rangle=\sin (\theta)|8\rangle-\sin (\theta)|1\rangle .
\end{aligned}
$$

This basis now appears less physically relevant than the flavor mixing angle $\phi$, which should probably be used instead. In any case they are trivially related, $\phi=\theta+\tan ^{-1}(\sqrt{2})$.

Now we will consider the quantum numbers of $q \bar{q}$ mesons in the nonrelativistic quark model, assuming that they are states of definite total quark spin $\mathrm{S}_{q \bar{q}}$ and orbital angular momentum $L_{q \bar{q}}$. Combining two spin-1/2 fermions gives total spin 0 and 1 ;

$$
2 \otimes 2= \begin{cases}3 & \left(\mathrm{~S}_{q \bar{q}}=1\right), \text { "spin triplets", } \\ \oplus & \\ 1 & \left(\mathrm{~S}_{q \bar{q}}=0\right), \text { "spin singlets". }\end{cases}
$$

These are combined with orbital wavefunctions to makes states of total $J, J_{z}$;

$$
\begin{gathered}
\left|q \bar{q}\left(J, J_{z}\right)\right\rangle=\sum_{L_{z}, S_{z}}<L, L_{z} ; S, S_{z}\left|J, J_{z}>\sum_{s_{z}, \bar{s}_{z}}<1 / 2, s_{z} ; 1 / 2, \bar{s}_{z}\right| S, S_{z}> \\
\cdot \int d \Omega Y_{\ell m}(\Omega) \cdot \int_{0}^{\infty} r^{2} d r \psi(r)\left|q_{s_{z}}(r / 2, \Omega), \bar{q}_{\bar{s}_{z}}(r / 2,-\Omega)\right\rangle .
\end{gathered}
$$

In the nonrelativistic quark model this is the state vector of a $q \bar{q}$ meson with a radial wavefunction $\psi(r)$, which is determined by the $q \bar{q}$ potential. This form assumes that the spin-dependent OGE tensor interaction is not present, since it mixes spin-triplet states such as ${ }^{3} \mathrm{~S}_{1}$ and ${ }^{3} \mathrm{D}_{1}$ that have $S=1, J=L \pm 1$. Given the quark-model meson state (30) we can easily derive quantum numbers. First, under parity $\mathcal{P}$ we transform $\Omega \rightarrow-\Omega$, which with the parity of $(-1)^{L}$ of the spherical harmonics and the intrinsic negative parity of a fermion-antifermion pair gives 


$$
\mathcal{P}|q \bar{q}(J, L, S)\rangle=(-1)^{L+1}|q \bar{q}(J, L, S)\rangle
$$

Another useful quantum number is C-parity; the $\mathcal{C}$ operator changes particles into antiparticles and vice versa, without changing any other state labels. Thus for example

$$
\mathcal{C}\left|u_{\uparrow} \bar{d}_{\downarrow}\right\rangle=\left|\bar{u}_{\uparrow} d_{\downarrow}\right\rangle
$$

If this operator is diagonal, the phase is called C-parity. Since $\mathcal{C}$ switches quarks and antiquarks, it is only diagonal on states with $q$ and $\bar{q}$ of the same flavor. Carrying out $\mathcal{C}$ on the $|q \bar{q}(J, L, S)\rangle$ states defined above, we find a phase of

$$
\mathcal{C}|q \bar{q}(J, L, S)\rangle=(-1)^{L+S}|q \bar{q}(J, L, S)\rangle
$$

C-parity is useful primarily because it is conserved in both QCD and QED, provided that we define the photon to have negative C-parity. Thus for example for the $\pi^{\circ}$, with $S=L=0$, $C=(+1)$ and therefore annihilation of a $\pi^{\circ}$ to only even numbers of photons is allowed. In contrast, for neutral vectors we have $C=(-1)^{L+S}=(-1)$, and for this reason the electromagnetic interaction of the $\rho^{o}$ is dominated by virtual transitions through a single photon. The strong reactions of single photons with light hadrons are often dominated by the reverse transition to a vector $q \bar{q}$ state, notably the $\rho^{\circ}$, which is known as "vector dominance".

A final useful multiplicative quantum number is G-parity. This is a combination of Cparity and isospin symmetry which is diagonal on charged states within an isomultiplet, and is also conserved in QCD in the limit of equal $u, d$ quark masses. G-parity combines $\mathrm{C}$ with a rotation by $\pi$ in isospin,

$$
\mathcal{G}=\mathcal{C} e^{i \pi I_{y}}
$$

C-parity on a $\left|\pi^{+}\right\rangle=-|u \bar{d}\rangle$, for example, gives a $(-1)^{L+S}|\bar{u} d\rangle=|d \bar{u}\rangle$ state, which is a $\left|\pi^{-}\right\rangle$; thus if applied to S-matrix elements, C-parity typically relates processes involving different asymptotic meson states. To obtain instead a diagonal operator $\mathcal{G}$ we follow this with a 
rotation about $I_{y}$, which from the theory of angular momentum gives a factor of $(-1)^{I}$. Thus we find for $u, d q \bar{q}$ isospin states in general

$$
\mathcal{G}|q \bar{q}(J, L, S, I)\rangle=C_{n}(-1)^{I}|q \bar{q}(J, L, S, I)\rangle=(-1)^{L+S+I}|q \bar{q}(J, L, S, I)\rangle,
$$

where $C_{n}$ is the C-parity of the neutral member of the multiplet. Since G-parity is diagonal on isospin states it can be used to exclude many a priori possible reactions. For example,

$$
\mathcal{G}|\pi\rangle=C_{n}(-1)^{I}|\pi\rangle=(-1)|\pi\rangle
$$

so any reaction which would scatter an even number of pions into an odd number is forbidden. Other examples are $\rho \not t \pi \pi$ (since $\rho$ has $(+1)$ G-parity), and $\omega \not \rightarrow \pi \pi$.

Finally we will discuss angular quantum numbers and state names. For spin-singlet $|q \bar{q}\rangle$ states we trivially have $J=L$ only, which in spectroscopic notation are ${ }^{1} L_{J=L}$. For spintriplet states we have $J=L+1, L, L-1$, and the states are called ${ }^{3} L_{J}$. In 1992 the Particle Data Group [2] standardized the names for states based on their quantum numbers, which for the flavor states we consider here, $q \bar{q}=u \bar{d}, u \bar{u}-d \bar{d}, d \bar{u} ; u \bar{u}+d \bar{d}, s \bar{s} ; c \bar{c}$, are as follows:

$$
\begin{cases}\pi ; \eta, \eta^{\prime} ; \eta_{c} & J^{P C}=\text { even }^{-+} \\ b ; h, h^{\prime} ; h_{c} & J^{P C}=\text { odd }^{+-} \\ \rho ; \omega, \phi ; \psi & J^{P C}=(\text { even or odd }, \geq 1)^{--} \\ a ; f, f^{\prime} ; \chi_{c} & J^{P C}=(\text { even or odd })^{++},\end{cases}
$$

and a subscript $J$ is added when necessary to avoid ambiguity. The $J^{P C}$ cases not listed are not allowed for $q \bar{q}$ states, and are known as "spin-parity exotics". These exotics are given the same name as a $q \bar{q}$ state which differs only in C-parity, thus for example and $I=1$, $J^{P C_{n}}=1^{-+}$exotic at $1900 \mathrm{MeV}$ would be named for the $1^{--} \rho$ as $\hat{\rho}(1900)$.

\section{B.' $q \bar{q}$ Mesons: Spectrum of States}

Having discussed the quantum numbers expected for $q \bar{q}$ states, we can now proceed to dynamical models of the spectrum of $q \bar{q}$ resonances. The most successful models for the description of spectroscopy are quark potential models, and in particular we shall consider the 
nonrelativistic quark potential model. The most widely used potential model for $q \bar{q}$ mesons is the relativized model of Godfrey and Isgur [3], and for $q q q$ baryons is the essentially equivalent model of Capstick and Isgur [4]. (Relativized versions differ from the nonrelativistic quark potential model only in relatively minor ways, such as the use of $H_{K E}=2 \sqrt{\vec{p}^{2}+m_{q}^{2}}$ as the kinetic term in place of $2 m_{q}+\vec{p}^{2} / m_{q}$, the retention of $m / E$ factors in matrix elements and the introduction of coordinate smearing in singular terms such as $\delta(\vec{r})$.)

. In the nonrelativistic quark potential model one typically assumes that the $q \bar{q}$ wavefunction in (30) is a solution of a Schrödinger equation with a potential that combines the color Coulomb term from one gluon exchange with a phenomenological linear confining term,

$$
\begin{gathered}
\left(H_{K E}+V\left(r_{q \bar{q}}\right)\right) \psi_{n}(r)=E_{n} \psi_{n}(r), \\
V(r)=-\frac{4}{3} \frac{\alpha_{s}}{r}+b r
\end{gathered}
$$

which is known as a "Coulomb plus linear" potential model. In addition at $O\left(v^{2} / c^{2}\right)$ in the quark motion one finds a series of spin-dependent terms from the nonrelativistic reduction of the Dirac spinor matrix elements. For the OGE interaction these are the Breit-Fermi Hamiltonian, familiar from atomic physics. The $O\left(v^{2} / c^{2}\right)$ spin-dependent interaction from the linear confining interaction, assuming it to be pure Lorentz scalar, is an inverted spinorbit term. In summary, the $O\left(v^{2} / c^{2}\right)$ spin-dependent $q \bar{q}$ Hamiltonian is

$$
\begin{gathered}
H_{\text {spin-dep. }}= \\
+\underbrace{\frac{32 \pi \alpha_{s}}{9 m_{q}^{2}} \vec{S}_{q} \cdot \vec{S}_{\bar{q}} \delta(\vec{x})}_{\text {OGE spin-spin }}+\underbrace{\frac{4 \alpha_{s}}{m_{q}^{2} r^{3}}\left(\vec{S}_{q} \cdot \hat{r} \vec{S}_{\bar{q}} \cdot \hat{r}-\frac{1}{3} \vec{S}_{q} \cdot \vec{S}_{\bar{q}}\right)}_{\text {OGE tensor }}+\underbrace{\frac{2 \alpha_{s}}{m_{q}^{2} r^{3}} \vec{L} \cdot \vec{S}}_{\text {OGE spin-orbit }}-\underbrace{\frac{b}{2 m_{q}^{2} r} \vec{L} \cdot \vec{S}}_{\text {scalar spin-orbit }} .
\end{gathered}
$$

Since these include singular terms such as $-1 / r^{3}$, they are usually treated perturbatively in the nonrelativistic Schrödinger formalism. As an example of a quarkonium spectrum predicted by this interaction we show the relatively straightforward $c \vec{c}$ system in Figure 2 . We shall use $c \bar{c}$ for our discussion of the Hamiltonian, because this spectrum is well established up to the second radial excitation. 
The qualitative effect of the various terms is as follows:

The OGE spin-spin force is a contact interaction (in the nonrelativistic limit only), so it causes a large positive splitting between the $L=0$ vector ${ }^{3} \mathrm{~S}_{1}$ and pseudoscalar ${ }^{1} \mathrm{~S}_{0}$ states, such as $\rho-\pi$ and $K^{*}-K$. Since the strength falls with $m_{q}$ as $1 / m_{q}^{2}$, it is much weaker at the $c \bar{c}$ mass scale, but still gives a $\psi-\eta_{c}$ splitting of about $110 \mathrm{MeV}$. In the Cornell potential model of $c a .1980$ [6] it was assumed that the confining interaction acted as the time-time components of Lorentz vectors $\left(\gamma_{0} \otimes \gamma_{0}\right)$ in Dirac spinor matrix elements, rather than as a scalar, $I \otimes I$. Such a vector-vector interaction at $O\left(v^{2} / c^{2}\right)$ leads to a spin-spin interaction of

$$
H_{\text {vectorspin-spin }}=+\frac{2}{3 m_{q}^{2}} \vec{S}_{q} \cdot \vec{S}_{\bar{q}} \nabla^{2} V(r)=\frac{4 b}{3 m_{q}^{2} r} .
$$

This vector confinement is now strongly ruled out by the discovery of the ${ }^{1} \mathrm{P}_{1} h_{c}$ spinsinglet $c \bar{c}$ charmonium state by the E760 Collaboration [7]; with typical $c \bar{c}$ potential model parameters, the vector confinement spin-spin term predicts a splitting between the c.o.g. of the $\left\{\chi_{j}\right\}{ }^{3} \mathrm{P}_{j}$ triplet states and the $h_{c}{ }^{1} \mathrm{P}_{1}$ singlet of $\approx 30 \mathrm{MeV}$, whereas experimentally the splitting is about $1 \mathrm{MeV}$. Scalar confinement expects a very small $h_{c}-\mathrm{P}_{\text {c.o.g }}$ splitting, because there is no scalar spin-spin interaction, and the (nonrelativistic) OGE spin-spin interaction is a contact term.

The spin-orbit interaction is quite interesting because the OGE and scalar confining terms have opposite signs. The OGE spin-orbit is shorter ranged, so we expect small- $L$ quarkonium states with sufficiently large masses to have an ordering of $M(J=L+1>L>L-1)$. For charmonium this is observed in the $\chi_{j}$ states. At sufficiently large $L$ the scalar spin-orbit should be largest, so multiplet inversion should take place. Models usually predict that this should happen by $L \sim 3$ for light $q \bar{q}$ systems [8], although it may be masked by mass shifts due to other interactions such as couplings to virtual two-meson channels [5].

Finally, there is a tensor OGE interaction, which modifies the characteristic $\vec{L} \cdot \vec{S}$ splitting pattern and mixes spin-triplet basis states related by $\Delta L=2$, for example ${ }^{3} \mathrm{~S}_{1} \leftrightarrow{ }^{3} \mathrm{D}_{1}$. The effect of the tensor term can be seen in the $c \bar{c}$ system in the $\chi_{j}$ states: In the ${ }^{3} \mathrm{P}_{j}$ and ${ }^{1} \mathrm{P}_{1}$ states the spin-orbit and tensor matrix elements are 


$$
\langle P|\vec{L} \cdot \vec{S}| P\rangle= \begin{cases}+1, & { }^{3} \mathrm{P}_{2} \\ 0, & { }^{1} \mathrm{P}_{1} \\ -1, & { }^{3} \mathrm{P}_{1} \\ -2, & { }^{3} \mathrm{P}_{0}\end{cases}
$$

and

$$
\langle P|T| P\rangle= \begin{cases}-1 / 30, & { }^{3} \mathrm{P}_{2} \\ 0, & { }^{1} \mathrm{P}_{1} \\ +1 / 6, & { }^{3} \mathrm{P}_{1} \\ -1 / 3, & { }^{3} \mathrm{P}_{0} .\end{cases}
$$

The masses reported for the corresponding $c \bar{c}$ states by the 1994 PDG are

$$
\langle P|T| P\rangle= \begin{cases}3556.17(13) \mathrm{MeV} & \chi_{2} \\ 3526.14(24) \mathrm{MeV} & h_{c} \\ 3510.53(12) \mathrm{MeV} & \chi_{1} \\ 3415.1(1.0) \mathrm{MeV} & \chi_{0} .\end{cases}
$$

Clearly the experimental masses are not well described by the equally-spaced spin-orbit pattern, whereas $\mathrm{P}$-wave mass splittings not far from experiment are observed when we include both spin-orbit and tensor terms with conventional parameter values (see Figure 2). We illustrate this in more detail in Figure 3 , which shows the spin-spin splitting of the $\psi-\eta_{c}$ and the splitting of the $\chi_{2}-\chi_{0}$ as we increase $\alpha_{s}$. (This is with a plausible charmed quark mass of $m_{c}=1.4 \mathrm{GeV}$ and a string tension of $b=0.18 \mathrm{GeV}^{2}$; this test is rather insensitive to $m_{c}$ and $b$, and these values are typical for $c \bar{c}$ spectroscopy.) The experimental S- and $\mathrm{P}$-multiplet splittings evidently require a value of $\alpha_{s}(c \bar{c}) \approx 0.4$, which is consistent with the value of $\alpha_{s}=0.45$ required to fit the spin-independent $\mathrm{P}_{c .0 . g}-\mathrm{S}_{\text {c.o.g }}$ of $\approx 456 \mathrm{MeV}$. This is a very nontrivial test of the validity of the OGE interaction in the $c \bar{c}$ spectrum, since it compares the leading order color Coulomb potential and the linear confining potential with the $O\left(v^{2} / c^{2}\right)$ spin-dependent interactions implied by OGE and scalar confinement.

Note that the value $\alpha_{s} \approx 0.4$ we need to describe $c \bar{c}$ spectroscopy is rather larger than the $\alpha_{s} \approx 0.2$ which was advocated long ago from fits to strong decay widths. For example, 
the Cornell collaboration [6] note that the $\psi$ total width would imply $\alpha_{s}=0.19(3)$ if we use the orthopositronium decay formula for ${ }^{3} \mathrm{~S}_{1}\left(e^{+} e^{-}\right) \rightarrow \gamma \gamma \gamma$ to model $\psi \rightarrow$ hadrons by $\psi \rightarrow g g g$. These tree-level nonrelativistic wavefunction-at-contact formulas are now known to have very important corrections from $O\left(\alpha_{s}\right)$ QCD radiative diagrams and especially from finite-range corrections to the contact approximation, so the small earlier value is presumably less reliable.

Now we shall begin our discussion of the light $q \bar{q}$ spectrum, as a preparation for our

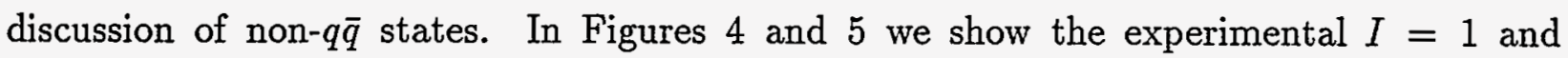
$I=0$ spectrum (shaded) and the predictions of Godfrey and Isgur [3] (lines). (The data represents the experimental situation in 1985.) Note that especially in $I=1$ there is quite good agreement between theory and experiment, and the missing levels are typically the lower-spin radial excitations or lower- $J$ members of multiplets, which are more difficult to extract from angular distributions. The largest discrepancy found since 1985 is the $a_{0}\left({ }^{3} \mathrm{P}_{0}\right)$ state, which was predicted to lie near $1.09 \mathrm{GeV}$, whereas the new Crystal Barrel candidate [9] is a much more massive $a_{0}(1450)$. Although the discrepancy is disturbing, it may not be a serious problem, because Godfrey and Isgur chose their parameters to give a rather light ${ }^{3} \mathrm{P}_{0} f_{0}(q \bar{q})$ state. Certainly the question of whether or not an $a_{0}(1450)$ state can be accommodated in the $q \bar{q}$ quark model should be reinvestigated, given what is now known about the importance of open-channel couplings for scalar $q \bar{q}$ states [5].

In the $I=0$ sector (Figure 5) the situation is much more complicated. Recent experimental work, carried out largely at LEAR, has added several new $f_{0}$ levels, and we now have six experimental $f_{0}$ resonances in the mass range between 980 and $1710 \mathrm{MeV}$. (These will be discussed in the following sections.) The quark model can perhaps accommodate three of these states as quarkonia, a ${ }^{3} \mathrm{P}_{0} q \bar{q}$, a ${ }^{3} \mathrm{P}_{0} s \bar{s}$ and a first radial excitation ${ }^{3} \mathrm{P}_{0}^{\prime} q \bar{q}$. Evidently there is much more to the resonance spectrum than just quarkonia, at least in the $f_{0}$ channel. In the following sections we review theoretical suggestions for what states other than $q \bar{q}$ might appear in the meson spectrum as resonances, and how these states might be distinguished from $q \bar{q}$. 


\section{Identification of $q \bar{q}$ States}

Traditionally meson resonances have been given $q \bar{q}$ quark model assignments based on their flavor and $J^{P C}$ quantum numbers, masses, and to a lesser extent their decay modes and production characteristics. This procedure works quite well in the mass region below about $1.5 \mathrm{GeV}$, where relatively few $q \bar{q}$ resonances are expected. As classic examples we might cite the tensor mesons $f_{2}(1270), a_{2}(1320)$ and $f_{2}^{\prime}(1525)$; the near degeneracy of the lighter $I=0$ and $I=1$ states suggests that they are nonstrange $u, d$ isospin partners, and the heavier $f_{2}^{\prime}(1525)$ might be an $s \bar{s}$ partner of these states. (Note the mass separation of $\approx 230 \mathrm{MeV}$, quite similar to $\phi-\omega \approx \phi-\rho \approx 240 \mathrm{MeV}$.) This is supported by the strong preference of the $f_{2}^{\prime}(1525)$ for $K \bar{K}$ final states over $\pi \pi$, as expected from the Zweig rule, which states that the initial $q \bar{q}$ pair in a meson (here $s \bar{s}$ ) cannot easily annihilate during a strong decay. In contrast, the lighter $f_{2}(1270)$ has comparable couplings to $\pi \pi$ and $K \bar{K}$. (The observed $f_{2}(1270) \rightarrow \pi \pi: K \bar{K}$ branching fraction ratio of $\approx 20: 1$ merely reflects the $P^{5}$ suppression expected for a D-wave decay.)

Unfortunately these simple ideas are not generally applicable to mesons in the mass region above $1.5 \mathrm{GeV}$. The complication is that many of the decay amplitudes of orbitally and (especially) radially excited states have nodes as functions of the outgoing hadron momentum, and if the physical momentum for a given mode is accidentally near a node, one could erroneously conclude that the coupling was weak for more fundamental reasons, such as flavor content. A simple example is the decay of the $f_{0}(q \bar{q})$ state to two pseudoscalars; in the "3 $\mathrm{P}_{0}$ " decay model of LeYaouanc et al. [10], with SHO wavefunctions the decay rate to $\pi \pi$ is given by [11]

$$
\Gamma\left(f_{0} \rightarrow \pi \pi\right)=\frac{2^{6}}{3^{3}} \gamma_{0}^{2} M_{f_{0}} \frac{P}{\beta}\left(1-\frac{2}{9} \frac{P^{2}}{\beta^{2}}\right) e^{-P^{2} / 6 \beta^{2}}
$$

Evidently this rate has a node at an outgoing pion momentum of $P=3 \beta / \sqrt{2}$, which is not far above the physical point; typically, decay models use $\beta \approx 0.4 \mathrm{GeV}$, which would give a vanishing width to $\pi \pi$ for an $f_{0}(q \vec{q})$ state with $M_{f_{0}}=1.6 \mathrm{GeV}$. In the $\approx 1.5-2.5 \mathrm{GeV}$ mass 
region which is of greatest interest in the search for non- $q \bar{q}$ mesons, the conventional $q \bar{q}$ states which we must identify as a background are orbital and radial excitations. Many of these will have complicated and counterintuitive decay amplitudes due to nodes. Resolution of this problem will require a careful investigation of radially and orbitally excited $q \bar{q}$ states, in particular their strong decay modes and branching fractions, so that we can test and improve the decay models. Given an accurate description of strong decays, it may be possible to identify all the $q \bar{q}$ levels in this mass region reliably.

Another approach, which has seen little application thus far, is to make use of electromagnetic couplings of meson resonances. One example is the two-photon couplings, which are measured in the reaction $e^{+} e^{-} \rightarrow e^{+} e^{-}+$hadrons; from a measurement of this cross section to a particular hadronic final state such as $\pi^{\circ} \pi^{\circ}$ one can see for example the $f_{2}(1270)$ as a clear bump, and the cross section implicitly determines the two-photon width times the branching fraction, here $\Gamma_{\gamma \gamma}\left(f_{2}\right) \cdot B\left(f_{2} \rightarrow \pi^{o} \pi^{\circ}\right)$. In principle even the absolute two-photon widths can be calculated from quark model wavefunctions, but for light $q \bar{q}$ mesons the results are rather sensitive to relativistic effects. (For $\Gamma_{\gamma \gamma}(c \bar{c})$ as an example see [12].)

The most convincing tests for $q \bar{q}$ candidates using electromagnetic couplings come from comparisons of the relative rates of a set of resonances with the same $J^{P C}$ which are possible members of a $u, d, s$ flavor multiplet. In $\gamma \gamma$ couplings, the decay amplitude involves the charge matrix element of two electromagnetic vertices,

$$
A(q \bar{q} \rightarrow \gamma \gamma) \propto\left\langle q \bar{q}\left|e_{q}^{2}\right| 0\right\rangle
$$

in a notation in which we suppress the photon state and current matrix elements. This gives relative amplitudes of

$$
\left\langle f: a: f^{\prime}\left|e_{q}^{2}\right| 0\right\rangle=\frac{(2 / 3)^{2}+(-1 / 3)^{2}}{\sqrt{2}}: \frac{(2 / 3)^{2}-(-1 / 3)^{2}}{\sqrt{2}}:(-1 / 3)^{2} .
$$

Given these relative amplitudes, the relative $\gamma \gamma$ decay rates for $I=0: I=1: s \bar{s}$ mesons of the same spatial state, neglecting phase space differences, are

$$
\Gamma_{\gamma \gamma}\left(f: a: f^{\prime}\right)=25: 9: 2 \text {. }
$$


In comparison, the measured two-photon widths of the corresponding tensor states are

$$
\Gamma_{\gamma \gamma}\left(f_{2}(1270): a_{2}(1320): f^{\prime}(1525)\right)=2.8(4) \mathrm{keV}: 1.04(9) \mathrm{keV}:: 0.105(17) \mathrm{keV}
$$

which clearly supports the $(u \bar{u} \pm d \bar{d}) / \sqrt{2}$ and $s \bar{s}$ assignments. (There is some additional suppression of the $s \bar{s}$ coupling to $\gamma \gamma$, due to the strange quark mass.) To date no radial excitations have been reported in $\gamma \gamma$, but once this data becomes available it should be very useful for identifying the members of $q \bar{q}$ flavor multiplets. Higher orbital excitations have already been reported, notably the $L=2 \pi_{2}(1670)$ and probably an $\eta_{2}$ state [13].

Finally, we note that single photon transitions, $(q \bar{q})_{i} \rightarrow \gamma(q \bar{q})_{f}$, would also be extremely useful for identifying $q \bar{q}$ states; these are more readily calculable theoretically, and also have a characteristic pattern of rates based on flavor,

$$
\Gamma\left((q \bar{q})_{i} \rightarrow \gamma(q \bar{q})_{f}\right)=9: 4: 1
$$

for

$$
\Delta I=1: s \vec{s}: \Delta I=0
$$

The $\Delta I=0,1$ ratios for transitions of $(u, d)$ meson pairs can be seen for example in decays of the light vectors,

$$
\Gamma(V \rightarrow \gamma \pi)= \begin{cases}720(40) & \mathrm{keV} \omega \rightarrow \pi^{o} \gamma \\ 68(7) & \mathrm{keV} \rho \rightarrow \pi \gamma .\end{cases}
$$

Although mesaurements of these radiative transitions would be very useful for the classification of higher $q \bar{q}$ and non- $q \bar{q}$ states, little work has been done along these lines. For example, of the many $f_{J}$ and $a_{J}$ states, only two transition rates to $(u, d)$ mesons have been measured, $a_{2} \rightarrow \pi \gamma=295(60) \mathrm{keV}$ and $a_{1} \rightarrow \pi \gamma=640(246) \mathrm{keV}$. Since measurements of radiative transitions could determine the nature of controversial states such as the $f_{1}(1420)$ (in $\gamma K \bar{K}$, to be discussed), they should certainly be carried out if possible. For $\pi \gamma$ modes this could use the inverse reaction (electroproduction) rather than photon emission, which has a large hadronic background. 


\section{NON-QQ̄ MESONS: INTRODUCTION}

The most exciting developments in QCD spectroscopy involve searches for resonances which are external to the conventional $q \bar{q}$ quark model of mesons. There are two general classes of such states, which are those with dominant gluonic excitations "gluonic hadrons" and states with more quarks and antiquarks than the familiar $q \bar{q}$ states.

Since QCD is a theory which contains both quarks and gluons as dynamical degrees of freedom, we would expect to see evidence of both these building blocks in the spectrum of physical color-singlet hadrons. It is remarkable, however, that of the hundreds of hadronic states now known, most can be described as states made only of quarks and antiquarks in the nonrelativistic quark model, and none of the remaining problematic resonances have been established as having dominant gluonic valence components. The best evidence for the presence of gluons at low energies is indirect, for example in the Breit-Fermi one-gluonexchange Hamiltonian used in potential models and in the $q \bar{q} \leftrightarrow s \bar{s}$ configuration mixing evident in the $\eta$ and $\eta^{\prime}$.

In addition to these gluonic states, one may also form color singlet combinations from multiquark systems of quarks and antiquarks, beginning with $q^{2} \bar{q}^{2}$. Although such resonances have been quite controversial, it now appears that such resonances do exist in nature, although they are dominantly "molecular" associations of meson pairs rather than single four-quark clusters.

Experimental studies now in progress may radically alter this situation, since there are now several resonances that, if confirmed, appear to be likely candidates for glueballs, hybrids and molecules. As we shall see, these states share several common features with theoretical expectations for these unusual hadronic states.

In this contribution we review current theoretical expectations for these gluonic hadrons and molecules, and briefly discuss some of the more recent and interesting experimental candidates for these states. 


\section{GLUEBALLS}

\section{A. Glueball masses}

A priori one would expect glueballs to be the most attractive gluonic hadrons experimentally, since they might be expected to differ most noticibly from $q \bar{q}$. In practice this naive expectation may not be realized; studies of the light glueball spectrum using lattice gauge theory have found that the lowest-lying glueball is a scalar, and its coupling to twopseudoscalar final states suggests a typical hadronic width. The next glueballs encountered at higher masses are predicted to be $0^{-+}$and $2^{++}$, and states which couple to two transverse gluons (presumably the lightest glueballs) do not contain exotic $J^{P C}$.

Although there have been many studies of the spectrum and quantum numbers expected for glueballs [14], the results of lattice gauge theory should be treated as the most relevant to experiment, since they bear the closest resemblance to full QCD. The assumptions of quenched lattice gauge theory are that decay channels do not modify glueball masses significantly (since the neglect of quarks implies stable light glueballs) and that the extrapolations to small lattice spacing and large lattive volume do not introduce important biases. If glueballs are not very broad objects, the assumption of stable glueballs should not introduce large errors in masses.

There are lattice predictions for the masses of glueballs with various $J^{P C}[15]$; the most reliable is presumably for the scalar glueball ground state, which is predicted to have a mass of

$$
\left(0^{++}\right)= \begin{cases}1.550(50) & \mathrm{GeV}[16] \\ 1.740(71) & \mathrm{GeV}[17]\end{cases}
$$

The corresponding mass estimate for the tensor glueballs is in the $2.2-2.4 \mathrm{GeV}$ range,

$$
\left(2^{++}\right)= \begin{cases}2.270(100) & \mathrm{GeV}[16] \\ 2.359(128) & \mathrm{GeV}[17] ;\end{cases}
$$

with the pseudoscalar glueball at a similar mass. 
There are obvious problems associated with the identification of a scalar state near 1.5 GeV. The $f_{0}$ sector is the most complicated of all meson sectors, with at least six problematical states, $f_{0}(980), f_{0}(1300), f_{0}(1365), f_{0}(1500) f_{0}(1590)$ and $f_{0}(1710)$. Since these are broad (except the $f_{0}(980)$ ) and overlapping resonances, the problem of identifying unusual states against the $q \bar{q}$ and $s \bar{s}$ background, and the related problems of separating individual resonances from interference and threshold effects are daunting ones. If the scalar glueball does have a typical hadronic width, as suggested by the work of Sexton et al. [18], it may be quite difficult to identify the scalar glueball convincingly. Amsler and Close [19] note that the near degeneracy of the pure LGT glueball and the $L=1 q \bar{q}$ and $s \bar{s}$ multiplets may lead to complicated mixing effects, so the physical states may be nontrivial combinations in flavor space, as in the $\eta$ and $\eta^{\prime}$ sector.

The tensor glueball may be an easier experimental target, since the expected mass is far above the lowest-lying $2^{++}$quarkonium states. Here the problem is that the mass region above $2 \mathrm{GeV}$ is poorly explored, so the problem of distinguishing a tensor glueball from the background of radial $-{ }^{3} \mathrm{P}_{2}$ and ${ }^{3} F_{2} q \bar{q}$ and $s \bar{s}$ states cannot yet be addressed. This lack of adequate information regarding the higher mass quarkonium spectrum is even more of a problem in the $0^{-+}$sector.

\section{B. Expectations for Glueball Properties}

Since we have no confirmed glueballs and the states predicted are in channels with a complicated or poorly explored resonance spectrum, it would be useful to have reliable theoretical predictions of glueball properties as a guide. The data we are likely to have on gluonic candidates in the near future are their strong branching fractions. Here a very characteristic naive glueball signature can be given, although it is easy to imagine ways in which this signature might be violated.

As gluons at the bare Lagrangian level have equal strength couplings to quarks of all flavors, one can make the assumption that flavor-symmetric couplings to hadron final states 
are approximately valid for physical glueballs. This gives a characteristic flavor-singlet branching fraction to pseudoscalar pairs, which is (neglecting phase space differences)

$$
\Gamma\left(G \rightarrow \pi \pi: K \bar{K}: \eta \eta: \eta \eta^{\prime}: \eta^{\prime} \eta^{\prime}\right) /(\text { phase space })=3: 4: 1: 0: 1 \text {. }
$$

Of course this simple pattern should at least incorporate the $|\vec{P}|$ from phase space for an S-wave decay, and there is in addition a decay form factor which depends on the unknown scalar glueball wavefunction and decay mechanism. Experience with the ${ }^{3} \mathrm{P}_{0} f_{0} q \bar{q}$ decay amplitude to $\pi \pi$, which has a node near the physical point [11], suggests that the naive pattern of flavor-singlet decay amplitudes may indeed be far from the physical couplings.

The accuracy of naive flavor-singlet couplings can be tested for a pure (quenched) scalar glueball in lattice gauge theory through a determination of the glueball-Ps-Ps three point function. Preliminary results for this coupling [18] indicate that flavor-singlet symmetry may indeed be badly violated at the amplitude level, and higher-mass Ps pairs are preferred in the decay. In view of the relatively large errors it will be important in future work to improve the statistics of this important lattice gauge theory measurement, and to extend these decay amplitude studies to the tensor and pseudoscalar glueball sectors.

In future experimental work it may be possible to determine or limit electromagnetic couplings of glueball candidates. Measurements of electromagnetic couplings of these resonances in one-photon $(R \rightarrow \gamma q \bar{q})$ and two-photon $(R \rightarrow \gamma \gamma)$ transitions would be extremely important for the clarification of the nature of these states; since theorists can calculate radiative transitions of $q \bar{q}$ states with reasonably accuracy, the radiative transition rates of a relatively pure glueball would clearly be anomalous relative to expectations for an $f_{0}(q \bar{q})$ state. If glueballs are indeed strongly mixed with $q \bar{q}$ and $s \bar{s}$ states, a convincing way to identify the flavor components of these mixed states would be through a comparison of the relative rates

$$
\Gamma\left(R \rightarrow \gamma \rho^{0}: \gamma \omega: \gamma \phi\right)
$$

since these act as flavor tags. Similarly, $\gamma \gamma$ couplings can be used to locate the scalar 
nonstrange $f_{0} q \vec{q}$ signal, since this state should have a strong coupling to $\gamma \gamma$. Results on this reaction have already been obtained by the Crystal Ball in the reaction $\gamma \gamma \rightarrow \pi^{\circ} \pi^{\circ}$.

Since a glueball should have suppressed couplings to $\gamma \gamma$, measurements of the $\gamma \gamma$ couplings of the various $f_{0}$ states and other light resonances would be very important contributions to light meson spectroscopy. This would be possible at $e^{+} e^{-}$facilities such as B-factories or a TCF.

\section{Summary of Glueball Candidates}

At present there are two prominent experimental candidates for glueballs, a scalar $f_{0}(1500)$ and the $\xi(2230)$, which is probably a tensor. The scalar candidate as reported by the Crystal Barrel [20] has a mass and width of

$$
M\left(f_{0}\right)=1520 \stackrel{+20}{-55} \mathrm{MeV}
$$

and

$$
\Gamma\left(f_{0}\right)=148 \underset{-25}{+20} \mathrm{MeV} .
$$

At this mass the $f_{0}(1500)$ cannot easily be assigned to a ${ }^{3} \mathrm{P}_{0}$ nonstrange $q \bar{q}$ state, but is consistent with the lower mass estimates from LGT for a scalar glueball. The width is also quite narrow for a ${ }^{3} \mathrm{P}_{0} q \bar{q}$ state at this mass. The decay pattern to pseudoscalar pairs is however inconsistent with flavor symmetry; the squared invariant couplings cited by Crystal Barrel $[20]$ are

$$
\begin{gathered}
\Gamma\left(f_{0}(1500) \rightarrow \pi \pi: K \bar{K}: \eta \eta: \eta \eta^{\prime}\right) /(\text { p.s. })= \\
1:<1 / 8.6(95 \% \text { c.l. }): 0.24 \pm 0.12: 0.35 \pm 0.15
\end{gathered}
$$

A priori this argues against a pure glueball interpretation, and subsequent work by Amsler and Close [20] has investigated the possibility that these decays may be consistent with a scalar glueball that has important $q \bar{q}$ and $s \bar{s}$ components, leading to an $\eta \eta^{\prime}$ mode and 
suppressing the $K \bar{K}$ mode. The limit on the coupling to $K \bar{K}$ is actually inferred from another experiment, and a more careful study of this coupling including interferences at the Crystal Barrel appears to find a much larger $K \bar{K}$ coupling [21]. This state may also have been seen in a recent reanalysis of the MarkIII data on $\psi \rightarrow \gamma \pi^{+} \pi^{-} \pi^{+} \pi^{-}$by Bugg et al. [22]; in this channel the $f_{0}(1500)$ appears dominantly in the " $\sigma \sigma$ " mode of two S-wave $\pi \pi$ pairs.

The second glueball candidate, which might be a narrow part of the $\xi(2230)$ previously reported by MarkIII in $\psi$ radiative decays, is reported by BES [23] to have very anomalous properties for a tensor above $2 \mathrm{GeV}$. The mass and width BES cite for this state in $K_{S} K_{S}$ are

$$
M(\xi)=2232{ }_{-7}^{+8} \pm 15 \mathrm{MeV}
$$

and

$$
\Gamma(\xi)=20 \stackrel{+25}{-16} \pm 10 \mathrm{MeV},
$$

with similar results in $P \bar{P}, K^{+} K^{-}$and $\pi^{+} \pi^{-}$. If this state is confirmed it is a remarkable discovery indeed. The mass is consistent with LGT expectations for the lightest tensor glueball (2), and the narrow width implies that this is certainly not a tensor quarkonium state. Since the couplings to $\pi \pi$ and $K \bar{K}$ appear to be approximately flavor symmetric, this does appear to be a natural glueball candidate.

Although Godfrey, Kokoski and Isgur [24] noted that the ${ }^{3} F_{2}$ and ${ }^{3} F_{4} s \bar{s}$ states expected near this mass could be relatively narrow, subsequent work by Blundell and Godfrey [25] has shown that other modes such as $K_{1}(1270) K$ are large, so $\Gamma\left(f_{2}(s \bar{s})\right) \geq 400 \mathrm{MeV}$. Similarly for the ${ }^{3} F_{4}$ Blundell and Godfrey now find a broader state given these additional modes, $\Gamma\left(f_{4}(s \bar{s})\right) \geq 130 \mathrm{MeV}$. Thus the $s \bar{s}$ assignments now appear implausible for an experimental width of $\leq 50 \mathrm{MeV}$.

There are several disturbing aspects of the experimental properties reported for this narrow effect. It has surprisingly small branching fractions to pseudoscalar pairs in view of 
the available phase space; branching fractions of a few percent are implied by the PS185 limit on $P \bar{P} \rightarrow \xi \rightarrow K \bar{K}$. A more important concern is that the reported statistical significance in each of the four channels studied by BES is rather small, $\approx 3 \sigma$. A caution is appropriate because many previously reported narrow states have been found to be artifacts of limited statistics (for example the $\zeta(8.3)$ ). In view of the remarkable properties reported for this state, measurements of these channels with higher statistics are extremely important tasks for any $e^{+} e^{-}$facility operating at the $\psi$ mass.

\section{HYBRIDS}

\section{A. Hybrid Quantum Numbers}

Hybrid mesons may be defined as resonances in which the dominant valence basis state is $q \tilde{q}$ combined with a gluonic excitation. Hybrids are attractive experimentally because, unlike glueballs, they span complete flavor nonets and hence provide many possibilities for experimental detection. In addition, the lightest hybrid multiplet is expected to include at least one $J^{P C}$-exotic (forbidden to $q \bar{q}$ ). For hybrids in In the MIT bag model [26], for example, the lightest gluon mode has $J^{P}=1^{+}$, so the lowest-lying $q \bar{q} g$ multiplet contains the quantum numbers

$$
J^{P C_{n}}(q \bar{q} g)= \begin{cases}0^{-+}, 1^{-+}, 2^{-+} & \left(S_{q \bar{q}}=1\right), \\ 1^{--} & \left(S_{q \bar{q}}=0\right) .\end{cases}
$$

The flux tube model extends this bag model list by adding a degenerate set with reversed $\{P, C\}$ to the lowest hybrid multiplet. Constituent gluon models of hybrids differ in that their lowest hybrid multiplet has P-wave $q \bar{q}$ quantum numbers [27] and so is nonexotic, although exotics appear in excited hybrid multiplets. An investigation of $q \bar{q} g$ interpolating fields [28] shows that hybrids can have any $J^{P C}$. 


\section{B. Hybrid Masses}

Hybrids have been studied in the literature using a wide range of models and techniques. These are the MIT bag model [26], constituent gluon models [27,29,30], the flux tube model [31-39], an adiabatic heavy-quark bag model [40] and heavy-quark lattice gauge theory [41]. Of the techniques now available to theorists for determining the properties of resonances in QCD, only Monte Carlo lattice gauge theory has not yet been applied. In all the approaches employed to date the lightest hybrids ( $H_{q}$, involving $u, d, s$ flavors) are predicted to have masses in the $\approx 1 \frac{1}{2}-2 \mathrm{GeV}$ region. A summary of hybrid mass predictions for the especially interesting $1^{-+}$exotic is given in the table below, taken from [37].

TABLE I. Predicted $1^{-+}$Hybrid Masses.

\begin{tabular}{llll}
\hline \hline state & mass $(\mathrm{GeV})$ & model & Ref. \\
\hline$H_{u, d}$ & $1.3-1.8$ & bag model & {$[26]$} \\
& $1.8-2.0$ & flux tube model & {$[31-34,37]$} \\
& $2.1-2.5$ & QCD sum rules (most after 1984) & {$[42-44]$} \\
& 2.1 & constituent gluon model & {$[30]$} \\
\hline$H_{c}$ & $\approx 3.9$ & adiabatic bag model & {$[40]$} \\
& $4.1-4.5$ & flux tube model & {$[32-34,37]$} \\
& $4.1-5.3$ & QCD sum rules (most after 1984) & {$[42-44]$} \\
& $4.19(3) \pm$ sys. & HQLGT & {$[41]$} \\
\hline$H_{b}$ & $10.49(20)$ & adiabatic bag model & {$[40]$} \\
& $10.8-11.1$ & flux tube model & {$[32-34]$} \\
& $10.6-11.2$ & QCD sum rules (most after 1984) & {$[42-44]$} \\
$10.81(3) \pm$ sys. & HQLGT & {$[41]$} \\
\hline \hline
\end{tabular}


A more detailed discussion of these predictions and the literature on hybrids is given by Barnes, Close and Swanson [37]; for other recent reviews of hybrids see [45].

Much of the recent interest in hybrids has derived from the predictions of the flux tube model, which gives rather precise predictions for masses and decay modes of hybrids. The original flux tube references [32-34] cited masses of $\approx 1.9 \mathrm{GeV}$ for the lightest $(q=u, d)$ hybrid multiplet, $\approx 4.3 \mathrm{GeV}$ for $c \bar{c}$ hybrids and $\approx 10.8 \mathrm{GeV}$ for $b \bar{b}$ hybrids. There is an overall variation of about $0.2-0.3 \mathrm{GeV}$ in these predictions, as indicated in Table I. Multiplet splittings are usually neglected in the flux tube model. This approximation may not be justified; a large inverted spin-orbit Thomas term for hybrids was found by Merlin and Paton [34].

A recent Hamiltonian Monte Carlo study [37] of the flux tube model determined hybrid masses without using the various questionable approximations of the early flux tube model studies, such as an adiabatic separation of quark and flux-tube motion and a small oscillation approximation for the flux tube. This Monte Carlo study generally confirmed the accuracy of the earlier flux-tube model mass estimates, for both $q \bar{q}$ and $c \bar{c}$ mesons (compared to experiment) and for hybrids (compared to the earlier approximate analytical calculations). These Monte Carlo predictions for light and charmonium levels are shown in Figs.5 and 6 of reference [37]. By varying the model parameters over a plausible range, this study concluded that the lightest hybrid masses in the flux tube model were

$$
\left(H_{u, d}\right)=1.8-1.9 \mathrm{GeV}
$$

for light quark hybrids and

$$
\left(H_{c}\right)=4.1-4.2 \mathrm{GeV}
$$

for charmonium hybrids. Excited hybrids were also considered, and the first hybrid orbital excitation $\left({ }_{\Lambda} L={ }_{1} \mathrm{D}\right)$ was found to appear at $2.30 \mathrm{GeV}, 400 \mathrm{MeV}$ above the lightest $\left({ }_{1} \mathrm{P}\right)$ hybrids. The same numerical result was found earlier by Merlin [35] using the adiabatic approximation. This ${ }_{1} \mathrm{D}$ multiplet contains the $J^{P C}$ states $(1,2,3)^{ \pm \mp}$ and $2^{ \pm \pm}$, which includes 
the exotics $1^{-+}, 2^{+-}$and $3^{-+}$. If the experimental candidates for ground-state hybrids near 1.8 GeV [46] and 1.6-2.2 GeV [47] are confirmed, it may be useful to search for members of the excited ${ }_{1} \mathrm{D}$ hybrid multiplet about $0.4 \mathrm{GeV}$ higher in mass.

\section{Light Hybrid Decay Modes}

Theoretical models predict rather characteristic two-body decay modes for hybrids. Both flux tube [36] and constituent gluon [29] models find that the lightest hybrids decay preferentially to pairs of one $L_{q \bar{q}}=0$ and one $L_{q \bar{q}}=1$ meson, for example $\pi f_{1}$ and $\pi b_{1}$. These unusual modes have received little experimental attention because they involve complicated final states, which may explain why hybrids were not been discovered previously. The original predictions for hybrid two-body modes given by Isgur, Kokoski and Paton [36] are given below in modern notation, together with information on secondary decay branches.

TABLE II. $J^{P C_{n}}$-Exotic Hybrid Meson Decays in the Flux Tube Model

\begin{tabular}{|c|c|c|c|c|c|c|c|}
\hline \multicolumn{5}{|c|}{ Hybrid Meson } & \multicolumn{3}{|c|}{ Secondary Decays } \\
\hline State & $\begin{array}{l}\text { IKP } \\
\text { label }\end{array}$ & $J^{P C_{n}}\left(I^{G}\right)$ & Decay & $\begin{array}{r}\Gamma_{H \rightarrow A B} \\
(\mathrm{MeV})\end{array}$ & $\begin{array}{r}\Gamma_{B} \\
(\mathrm{MeV})\end{array}$ & Decay & b.f. \\
\hline$\hat{a}_{2}(1900)$ & $x_{2}^{+-}$ & $2^{+-}\left(1^{+}\right)$ & {$\left[\pi a_{2}(1320)\right]_{P}$} & 450 & 103 & $\begin{array}{l}a_{2} \rightarrow \rho \pi \\
\rightarrow \eta \pi \\
\rightarrow \omega \pi \pi \\
\rightarrow K \tilde{K}\end{array}$ & $\begin{array}{r}70 \% \\
15 \% \\
11 \% \\
5 \%\end{array}$ \\
\hline & & & {$\left[\pi a_{1}(1260)\right]_{P}$} & 100 & $\sim 400$ & $a_{1} \rightarrow \rho \pi$ & most \\
\hline & & & {$\left[\pi h_{1}(1170)\right]_{P}$} & 150 & 360 & $h_{1} \rightarrow \rho \pi$ & seen \\
\hline$\hat{f}_{2}(1900)$ & $y_{2}^{+-}$ & $2^{+-}\left(0^{-}\right)$ & {$\left[\pi b_{1}(1235)\right]_{P}$} & 500 & 155 & $\begin{array}{l}b_{1} \rightarrow(\omega \pi)_{S, D} \\
\rightarrow \eta \rho\end{array}$ & $\begin{array}{r}\text { most } \\
\text { seen }\end{array}$ \\
\hline$\hat{f}_{2}^{\prime}(2100)$ & $z_{2}^{+-}$ & $2^{+-}\left(0^{-}\right)$ & {$\left[K K_{2}^{*}(1430)\right]_{P}$} & 250 & 98 & $\begin{array}{l}K_{2}^{*} \rightarrow K \pi \\
\rightarrow K^{*} \pi\end{array}$ & $\begin{array}{l}50 \% \\
25 \%\end{array}$ \\
\hline
\end{tabular}




\begin{tabular}{|c|c|c|c|c|c|c|c|}
\hline & & & {$\left[K K_{1}(1400)\right]_{P}$} & 200 & 174 & $\begin{array}{l}\rightarrow K^{*} \pi \pi \\
K_{1} \rightarrow K^{*} \pi\end{array}$ & $\begin{array}{l}13 \% \\
94 \%\end{array}$ \\
\hline \multirow[t]{4}{*}{$\hat{\rho}(1900)$} & $x_{1}^{-+}$ & $1^{-+}\left(1^{-}\right)$ & {$\left[\pi b_{1}(1235)\right]_{S, D}$} & 100,30 & 155 & $b_{1} \rightarrow(\omega \pi)_{S, D}$ & most \\
\hline & & & {$\left[\pi f_{1}(1285)\right]_{S, D}$} & 30,20 & 24 & $f_{1} \rightarrow \eta \pi \pi$ & $50 \%$ \\
\hline & & & & & & $\rightarrow 4 \pi(\rho \pi \pi)$ & $38 \%$ \\
\hline & & & & & & $\rightarrow a_{0}(980) \pi$ & $37 \%$ \\
\hline \multirow[t]{3}{*}{$\hat{\omega}(1900)$} & $y_{1}^{-+}$ & $1^{-+}\left(0^{+}\right)$ & {$\left[\pi a_{1}(1260)\right]_{S, D}$} & 100,70 & $\sim 400$ & $a_{1} \rightarrow \rho \pi$ & most \\
\hline & & & {$[\pi \pi(1300)]_{P}$} & 100 & $200-600$ & $\pi(1300) \rightarrow \rho \pi$ & seen \\
\hline & & & {$\left[K K_{1}(1400)\right]_{S}$} & 100 & 174 & $K_{1} \rightarrow K^{*} \pi$ & $94 \%$ \\
\hline \multirow[t]{6}{*}{$\hat{\phi}(2100)$} & $z_{1}^{-+}$ & $1^{-+}\left(0^{+}\right)$ & {$\left[K K_{1}(1270)\right]_{D}$} & 80 & 90 & $K_{1} \rightarrow K \rho$ & $42 \%$ \\
\hline & & & & & & $\rightarrow K_{0}^{*} \pi$ & $28 \%$ \\
\hline & & & & & & $\rightarrow K^{*} \pi$ & $16 \%$ \\
\hline & & & & & & $\rightarrow K \omega$ & $11 \%$ \\
\hline & & & {$\left[K K_{1}(1400)\right]_{S}$} & 250 & 174 & $K_{1} \rightarrow K^{*} \pi$ & $94 \%$ \\
\hline & & & {$[K K(1460)]_{P}$} & 30 & 250 & $K \rightarrow K \pi \pi$ & seen \\
\hline \multirow[t]{3}{*}{$\hat{a}_{0}(1900)$} & $x_{0}^{+-}$ & $0^{+-}\left(1^{+}\right)$ & {$\left[\pi a_{1}(1260)\right]_{P}$} & 800 & $\sim 400$ & $a_{1} \rightarrow \rho \pi$ & nost \\
\hline & & & {$\left[\pi h_{1}(1170)\right]_{P}$} & 100 & 360 & $h_{1} \rightarrow \rho \pi$ & seen \\
\hline & & & {$[\pi \pi(1300)]_{S}$} & 900 & $200-600$ & $\pi(1300) \rightarrow \rho \pi$ & seen \\
\hline \multirow[t]{2}{*}{$\hat{f}_{0}(1900)$} & $y_{0}^{+-}$ & $0^{+-}\left(0^{-}\right)$ & {$\left[\pi b_{1}(1235)\right]_{P}$} & 250 & 155 & $b_{1} \rightarrow(\omega \pi)_{S, D}$ & aost \\
\hline & & & & & & $\rightarrow \eta \rho$ & seen \\
\hline \multirow[t]{3}{*}{$\hat{f}_{0}^{\prime}(2100)$} & $z_{0}^{+-}$ & $0^{+-}\left(0^{-}\right)$ & {$\left[K K_{1}(1270)\right]_{P}$} & 800 & 90 & See $\hat{\phi}(2100)$ & \\
\hline & & & {$\left[K K_{1}(1400)\right]_{P}$} & 50 & 174 & $K_{1} \rightarrow K^{*} \pi$ & $94 \%$ \\
\hline & & & {$[K K(1460)]_{S}$} & 800 & 250 & $K \rightarrow K \pi \pi$ & \\
\hline
\end{tabular}


These decay predictions are quite interesting because they suggest that many hybrids are so broad that they will be effectively invisible, whereas a few hybrids should be narrow enough to be easily observable in certain channels. The $I=1 J^{P C}=1^{-+}$exotic had already been cited as an attractive experimental candidate, and this work suggested that this state should be relatively narrow for a hybrid $\Gamma_{\text {tot }} \approx 200 \mathrm{MeV}$, and that the $S+P$ modes $\pi b_{1}$ and $\pi f_{1}$ should be the dominant final states populated by decays of this state. This work has motivated several studies of $\pi b_{1}$ and $\pi f_{1}$ final states, which show possible indications of resonant amplitudes in $1^{-+}$.

These original flux tube decay calculations were for the three exotic $J^{P C}$ quantum numbers in the lowest flux-tube multiplet. Since this multiplet contains a total of eight $J^{P C}$ assignments, $1^{ \pm \pm}$(for $\mathrm{S}_{q \bar{q}}=0$ ) and $2^{ \pm \mp} ; 1^{ \pm \mp} ; 0^{ \pm \mp} ;\left(\right.$ for $\mathrm{S}_{q \bar{q}}=1$ ), one might wonder whether the nonexotic hybrids might be narrow enough to be observable. The decay amplitudes of these nonexotic hybrids were recently calculated by Close and Page [38], who also checked the exotic decay amplitudes and found reasonable agreement with Isgur, Kokoski and Paton. The Close-Page results for decays of $(u \bar{u} \pm d \bar{d}) / \sqrt{2}$ hybrids are reproduced below, since they suggest some very important experimental tests of the flux tube model.

Close and Page predict that many of the nonexotic hybrids are also so broad as to be effectively unobservable. There are two striking exceptions. One is a $1^{--} \omega$-hybrid with a total width of only $\approx 100 \mathrm{MeV}$, which decays to $K_{1}(1270) K$ and $K_{1}(1400) K$; this should be searched for in $K_{1} K$ final states, perhaps in photoproduction. A second interesting nonexotic hybrid is a $\pi_{2}$, with $\Gamma_{\text {tot }} \approx 170 \mathrm{MeV}$. This may be the high-mass $\pi_{2}$ state which has been reported repeatedly in photoproduction reactions at a mass near $1775 \mathrm{MeV}$ [46]. Other notable conclusions are that 1) several other hybrids, including exotics, have total widths near $300 \mathrm{MeV}$ and so should be observable, and 2) the $I=00^{+-}$exotic found by Isgur et al. to have $\Gamma_{b_{1} \pi}=250 \mathrm{MeV}$ actually has very large $K_{1} K$ modes and so should be unobservable.

In addition Close and Page investigate the "forbidden" decay modes such as $\hat{\rho}(1900) \rightarrow$ $\rho \pi$, and find that, due to differences in the $\rho$ and $\pi$ spatial wavefunctions, these S+S modes 
are present with partial widths of typically $\sim 10 \mathrm{MeV}$. Thus it is interesting to search relatively straightforward modes such as $\rho \pi$ for hybrids, in addition to the favored but more difficult $\mathrm{S}+\mathrm{P}$ modes such as $b_{1} \pi, f_{1} \pi$ and $K_{1} K$.

TABLE III. Flux tube results for the dominant decay modes (in $\mathrm{MeV}$ ) of $I=1$ hybrids, from Close and Page [38].

\begin{tabular}{|c|c|c|c|c|c|c|c|c|c|c|c|}
\hline$A$ & $B, C$ & $L$ & $\Gamma$ & $A$ & $B, C$ & $L$ & $\Gamma$ & $A$ & $B, C$ & $L$ & $\Gamma$ \\
\hline \multirow[t]{5}{*}{$2^{-+}$} & $f_{2}(1270) \pi$ & $\mathrm{S}$ & 40 & \multirow[t]{7}{*}{$1^{+-}$} & $a_{2}(1320) \pi$ & $\mathrm{P}$ & 175 & \multirow[t]{7}{*}{$1^{-+}$} & $f_{1}(1285) \pi$ & $\mathrm{S}$ & 40 \\
\hline & & $\mathrm{D}$ & 20 & & $a_{1}(1260) / \pi$ & $\mathrm{P}$ & 90 & & & $\mathrm{D}$ & 20 \\
\hline & $b_{1}(1235) \pi$ & $\mathrm{D}$ & 40 & & $h_{1}(1170) \pi$ & $\mathrm{P}$ & 175 & & $b_{1}(1235) \pi$ & $\mathrm{S}$ & 150 \\
\hline & $a_{2}(1320) \eta$ & $\mathrm{S}$ & $\sim 40$ & & $b_{1}(1235) \eta$ & $\mathrm{P}$ & 150 & & & $\mathrm{D}$ & 20 \\
\hline & $K_{2}^{*}(1430) K$ & $\mathrm{~S}$ & $\sim 30$ & & $K_{2}^{*}(1430) K$ & $\mathrm{P}$ & 60 & & $a_{1}(1260) \eta$ & $\mathrm{S}$ & 50 \\
\hline \multirow[t]{4}{*}{$2^{+-}$} & $a_{2}(1320) \pi$ & $\mathrm{P}$ & 200 & & $K_{1}(1270) K$ & $\mathrm{P}$ & 250 & & $K_{1}(1270) K$ & $\mathrm{~S}$ & 20 \\
\hline & $a_{1}(1260) \pi$ & $\mathrm{P}$ & 70 & & $K_{0}^{*}(1430) K$ & $\mathrm{P}$ & 70 & & $K_{1}(1400) K$ & $S$ & $\sim 125$ \\
\hline & $h_{1}(1170) \pi$ & $\mathrm{P}$ & 90 & \multirow[t]{8}{*}{$1^{++}$} & $f_{2}(1270) \pi$ & $\mathrm{P}$ & 175 & \multirow[t]{3}{*}{$0^{-+}$} & $f_{2}(1270) \pi$ & $\mathrm{D}$ & 20 \\
\hline & $b_{1}(1235) \eta$ & $\mathrm{P}$ & $\sim 15$ & & $f_{1}(1285) \pi$ & $\mathrm{P}$ & 150 & & $f_{0}(1300) \pi$ & $S$ & $\sim 150$ \\
\hline \multirow[t]{6}{*}{$0^{+-}$} & $a_{1}(1260) \pi$ & $\mathrm{P}$ & 700 & & $f_{0}(1300) \pi$ & $\mathrm{P}$ & $\sim 20$ & & $K_{0}^{*}(1430) K$ & $\mathrm{~s}$ & $\sim 200$ \\
\hline & $h_{1}(1170) \pi$ & $\mathrm{P}$ & 125 & & $a_{2}(1320) \eta$ & $\mathrm{P}$ & 50 & \multirow[t]{5}{*}{$1^{--}$} & $a_{2}(1320) \pi$ & $\mathrm{D}$ & 50 \\
\hline & $b_{1}(1235) \eta$ & $P$ & 80 & & $a_{1}(1260) \eta$ & $\mathrm{P}$ & 90 & & $a_{1}(1260) \pi$ & $\mathrm{s}$ & 150 \\
\hline & $K_{1}(1270) K$ & $\mathrm{P}$ & 600 & & $K_{2}^{*}(1430) K$ & $\mathrm{P}$ & $\sim 20$ & & & $\mathrm{D}$ & 20 \\
\hline & $K_{1}(1400) K$ & $\mathrm{P}$ & 150 & & $K_{1}(1270) K$ & $\mathrm{P}$ & 40 & & $K_{1}(1270) K$ & $\mathrm{~s}$ & 40 \\
\hline & & & & & $K_{1}(1400) K$ & $\mathrm{P}$ & $\sim 20$ & & $K_{1}(1400) K$ & $\mathrm{~S}$ & $\sim 60$ \\
\hline
\end{tabular}


TABLE IV. As in Table III, for $I=0$ nonstrange hybrids.

\begin{tabular}{|c|c|c|c|c|c|c|c|c|c|c|c|}
\hline$A$ & $B, C$ & $L$ & $\Gamma$ & $A$ & $B, C$ & $L$ & $\Gamma$ & $A$ & $B, C$ & $L$ & $\Gamma$ \\
\hline \multirow[t]{4}{*}{$2^{-+}$} & $a_{2}(1320) \pi$ & $\mathrm{S}$ & 125 & $2^{+-}$ & $b_{1}(1235) \pi$ & $\mathrm{P}$ & 250 & \multirow[t]{7}{*}{$1^{++}$} & $a_{2}(1320) \pi$ & $\mathrm{P}$ & 500 \\
\hline & & $\mathrm{D}$ & 60 & & $h_{1}(1170) \eta$ & $\mathrm{P}$ & 30 & & $a_{1}(1260) \pi$ & $\mathrm{P}$ & 450 \\
\hline & $f_{2}(1270) \eta$ & $\mathrm{S}$ & $\sim 50$ & $0^{+-}$ & $b_{1}(1235) \pi$ & $\mathrm{P}$ & 300 & & $f_{2}(1270) \eta$ & $\mathrm{P}$ & 70 \\
\hline & $K_{2}^{*}(1430) K$ & $\mathrm{~S}$ & $\sim 30$ & & $h_{1}(1170) \eta$ & $\mathrm{P}$ & 90 & & $f_{1}(1285) \eta$ & $\mathrm{P}$ & 60 \\
\hline \multirow[t]{5}{*}{$1^{+-}$} & $b_{1}(1235) \pi$ & $\mathrm{P}$ & 500 & & $K_{1}(1270) K$ & $\mathrm{P}$ & 600 & & $K_{2}^{*}(1430) K$ & $\mathrm{P}$ & $\sim 20$ \\
\hline & $h_{1}(1170) \eta$ & $\mathrm{P}$ & 175 & & $K_{1}(1400) K$ & $\mathrm{P}$ & 150 & & $K_{1}(1270) K$ & $\mathrm{P}$ & 40 \\
\hline & $K_{2}^{*}(1430) K$ & $\mathrm{P}$ & 60 & $1^{-+}$ & $a_{1}(1260) \pi$ & $\mathrm{S}$ & 100 & & $K_{1}(1400) K$ & $\mathrm{P}$ & $\sim 20$ \\
\hline & $K_{1}(1270) K$ & $\mathrm{p}$ & 250 & & & $\mathrm{D}$ & 70 & \multirow[t]{4}{*}{$0^{-+}$} & \multirow{4}{*}{$\begin{array}{l}a_{2}(1320) \pi \\
f_{0}(1300) \eta \\
K_{0}^{*}(1430) K\end{array}$} & \multirow{4}{*}{$\mathrm{S}$} & \multirow{4}{*}{$\begin{array}{r}60 \\
\sim 200 \\
\sim 200\end{array}$} \\
\hline & $K_{0}^{*}(1430) K$ & $\mathrm{P}$ & 70 & & $f_{1}(1285) \eta$ & $\mathrm{S}$ & 50 & & & & \\
\hline \multirow[t]{2}{*}{$1^{--}$} & $K_{1}(1270) K$ & $\mathrm{~S}$ & 40 & & $K_{1}(1270) K$ & $\mathrm{~S}$ & 20 & & & & \\
\hline & $K_{1}(1400) K$ & $\mathrm{~S}$ & 60 & & $K_{1}(1400) K$ & $\mathrm{~S}$ & $\sim 125$ & & & & \\
\hline
\end{tabular}




\section{Prospects for Charmonium Hybrids}

The predictions of the flux tube model and heavy-quark LGT (Table I) that hybrid charmonium states should exist at 4.1-4.2 GeV are expecially interesting. Charmonium spectroscopy is rather well understood up to about $3.8 \mathrm{GeV}$, so searches for unusual states should be straightforward near this mass. Since only a few open charm channels occur below 4.3 GeV, for a considerable range of hybrid masses one might anticipate rather narrow hybrid resonances. This possibility receives additional support from the flux tube model $[36,38]$, which predicts that the dominant two body decay modes of the lowest lying hybrids are an $L=0$ and $L=1 q \bar{q}$ meson pair. These $S+P$ thresholds are rather high in mass, about 4.3 GeV for $c \bar{c}$ hybrids and $11.0 \mathrm{GeV}$ for $b \bar{b}$ hybrids. The possibility that relatively narrow hybrid charmonium states may exist within this $3.8-4.3 \mathrm{GeV}$ window provides an exciting opportunity for $e^{+} e^{-}$facilities such as BEPC, KEK and a Tau-Charm Factory.

Calculations of the decay widths of charmonium hybrids have been carried out in the flux tube model by Close and Page [48], assuming masses of $\approx 4.1-4.2 \mathrm{GeV}$. These partial widths (to $D^{*} D$ ) are found to be quite small, typically only $\sim 1-10 \mathrm{MeV}$. Thus if there are relatively unmixed charmonium hybrids, the $1^{--}$vector hybrids should appear as narrow spikes in $R$ in this mass range. For this reason a detailed scan of $R$ starting near the open charm threshold would be a first priority at a Tau-Charm Factory. Close and Page speculate about a more complicated possibility, which is that the $\psi(4040)$ and $\psi(4160)$ may be equal-weight linear combinations of $3 S|c \bar{c}\rangle$ and the $1^{--} c \bar{c}$-hybrid. (The usual assignment is that the $\psi(4040)$ is $3 S$ and the $\psi(4160)$ is $2 \mathrm{D}$ [49].) The Close-Page linear combinations would explain why the $e^{+} e^{-}$widths are approximately equal and relatively large for both states, which is surprising if one is a $\mathrm{D}$-wave $c \bar{c}$. The assignments for the $\psi$ states above open-charm thresholds can be tested by measurements of their branching fractions to $D D, D^{*} D, \ldots, D_{s}^{*} D_{s}^{*}$. The branching fractions predicted by these models are very characteristic; unfortunately but they have not yet been measured accurately. Determination of these branching fractions would be a high priority at an $e^{+} e^{-}$machine operating at the $\psi$ mass. 
Finally, we note that the non-vector hybrids can also be produced in $e^{+} e^{-}$through a "continuum cascade", as suggested by D.Bugg, and discussed in references $[49,50]$. In this. approach one produces a high-mass $c \bar{c}$ system in the continuum, perhaps at $5 \mathrm{GeV}$; this may then decay hadronically to hybrid charmonium levels of various $J^{P C}$, and the hybrid in turn decays hadronically to a characteristic state such as the $\psi$. Thus one can search for the decay chain

$$
e^{+} e^{-} \rightarrow c \bar{c} \rightarrow H_{c} \eta ; \quad H_{c} \rightarrow \eta \psi ; \quad \psi \rightarrow e^{+} e^{-}
$$

in the final state $\eta \eta e^{+} e^{-}$, triggering on a lepton pair from the $\psi$ and $\gamma \gamma$ pairs from the two $\eta$ s. The $\eta \psi$ invariant mass distribution can then be studied for evidence of hybrids or $c \bar{c}$ states. Other quantum numbers can be investigated by replacing $\eta$ by other hadrons, by $(\pi \pi)_{S}$ for example, in the hadronic cascades.

\section{E. Hybrid Experimental Candidates}

There are several experimental candidates for hybrids, but as in the case of glueballs there are no generally accepted states at present.

In the exotic channels (which would be most convincing evidence for a hybrid), previous claims by GAMS that a resonant signal had been detected in the $1^{-+}$wave of $\eta \pi$ [51] have now been withdrawn. A KEK experiment [52] instead finds evidence for a resonant $1^{-+} \eta \pi$ wave but with the mass and width of the $a_{2}(1320)$; this surprising result obviously must be checked carefully for "feedthrough" of the $a_{2}$ amplitude. VES [53] has studied $\pi \eta$ and $\pi \eta^{\prime}$ and report a broad, higher-mass effect in $\pi \eta$ and especially in $\pi \eta^{\prime}$, with a mass near 1.6 $\mathrm{GeV}$. The phase motion of the ${1^{-+}}^{-}$component has not yet been determined.

Studies of the final state $f_{1} \pi$ suggested by flux tube model decay calculations are underway $[53,47]$, and preliminary evidence for a possible $1^{-+}$signal has been reported by E818 at BNL [47].

There have been several observations of a photoproduced $I=1$ state at about $1775 \mathrm{MeV}$ in $\rho \pi$ and $f_{2} \pi$ [46], which is too heavy to be the $\pi_{2}(1670)$ without complicated interference 
effects. Although the quantum numbers of this state have not been determined definitively, $1^{-+}$is preferred over $2^{-+}$. A possible narrow $1^{-+}$state has been reported by GAMS in $\eta \eta^{\prime}$ at a mass of $1910 \mathrm{MeV}$; here there are rather few events, so it will be important to improve the statistics. Several experiments plan future studies of these channels, including E818 (to study $\pi^{-} f_{1}$ ) [54] and E852 (to study $\pi f_{1}$ and $\pi \eta$ ) [55] at BNL.

In addition to exotic hybrid candidates there are several nonexotic candidates; recall for example the Close-Page result that a hybrid with $\pi_{2}$ quantum numbers is expected to be relatively narrow, and should be visible in $f_{2} \pi$. One way to distinguish hybrids from $q \bar{q}$ spin-singlet states is through their strong branching fractions; a selection rule in the ${ }^{3} \mathrm{P}_{0}$ decay model forbids the decay of a spin-singlet $q \bar{q}$ state to two final spin-singlet quarkonia,

$$
(q \bar{q})_{S=0} \not \nrightarrow(q \bar{q})_{S=0}+(q \bar{q})_{S=0} .
$$

In the $\pi_{2}$ channel this selection rule forbids the decay of a ${ }^{1} \mathrm{D}_{2} q \bar{q} \pi_{2}$ to a ${ }^{1} \mathrm{P}_{1} b_{1}$ plus a ${ }^{1} \mathrm{~S}_{0} \pi$,

$$
\pi_{2}(q \bar{q}) \not b_{1} \pi
$$

but allows it for a hybrid $\pi_{2}$ which does not have the $q \bar{q}$ pair in an $S=0$ configuration. Close and Page find the $b_{1} \pi$ mode of a $\pi_{2}$ hybrid should be rather large, so it is especially important to search the $b_{1} \pi$ channel for evidence of a $2^{-+}$hybrid signal. The Crystal Barrel collaboration has preliminary results for $\pi b_{1}$ but at present sees no evidence for unusual states [56].

Other nonexotic hybrid candidates which have been suggested recently are a $\pi(1800)$ reported by VES [57] and the nonstrange $1^{--}$states near $1.4-1.7 \mathrm{GeV}[58]$. The $\pi(1800)$ is cited as a possible hybrid because it has unusual branching fractions, including a significant coupling to $\pi \eta \eta$, apparently through the glueball candidate $f_{0}(1500) \rightarrow \eta \eta$. This $\pi(1800)$ is also reported by VES in $\omega \rho, a_{0}(980) \eta, \pi f_{0}(980)$ and $\pi f_{0}(1300)$. The decay mode $\pi(1800) \rightarrow$ $\pi \rho$ is notably absent, and $f_{2} \pi$ is also weak or absent.

Although the weakness of the $\rho \pi \mathrm{S}+\mathrm{S}$ mode is indeed suggestive of a hybrid, since a $\pi(1800)$ is expected in the quark potential model as a second radial excitation, one should be 
careful to exclude this possibility. Radial quarkonia can have unusual branching fractions due to the nodes in their decay amplitudes, and in the ${ }^{3} \mathrm{P}_{0}$ decay model with SHO wavefunctions the amplitude for $\pi(3 S) \rightarrow \rho \pi$ actually has a node at $M=1.88 \mathrm{GeV}$ for $\beta=0.35 \mathrm{GeV}$, so the weakness of the $\rho \pi$ mode is understandable for a $3 S$ state. However the same model predicts a weak $f_{0}(1300) \pi$ mode which disagrees with experiment. The decay amplitude for $\pi(3 S) \rightarrow \rho(2 S) \pi$ is predicted to be quite large, so a search for a $\rho(1450) \pi$ final state would be useful.

Similarly the unusual properties of the nonstrange $I=0$ and $I=1$ vectors near $1.5 \mathrm{GeV}$ have led to suggestions that hybrid vector states may be present near this mass $[58,59]$. In $I=1$, for example, the two states $\rho(1450)$ and $\rho(1700)$ are usually assigned to $2^{3} \mathrm{~S}_{1}$ and ${ }^{3} \mathrm{D}_{1}$ respectively, but the very large $\rho(1450) \rightarrow 2\left(\pi^{+} \pi^{-}\right)$mode [59] is in conflict with quark model expectations for a $2^{3} \mathrm{~S}_{1}$ state [59-61]. A better understanding of these vector states would be possible with a detailed isobar analysis of their quasi two-body strong decay modes.

As a general conclusion regarding nonexotic hybrids, these comparisons of strong decay modes illustrate the importance of having an accurate understanding of the decays of radially excited $q \bar{q}$ states. Both experimental and theoretical studies of the strong decays of radially excited $q \bar{q}$ candidates such as the $\pi(1300), \rho(1450), \phi(1680)$ and so forth will be required if we are to distinguish $q \bar{q}$ from non- $q \bar{q}$ glueballs and hybrids with identical quantum numbers.

\section{MOLECULES}

\section{A. Introduction}

Here we consider the experimental and theoretical status of hadronic molecules, which are weakly-bound states of two or more hadrons. We begin with a brief history of the subject of multiquark systems, following which we suggest some signatures for molecules and review experimental candidates. A more general understanding of $2 \rightarrow 2$ hadron-hadron scattering amplitudes will be crucial for molecule searches, since bound states are a special feature of 
the more general problem of determining hadron-hadron scattering amplitudes. We conclude with a short summary of some recent theoretical work on determining scattering amplitudes from the quark model.

In the 1970s it was widely believed that there would be a very rich spectrum of discrete levels of multiquark resonances. The argument was that the many known $q \bar{q}$ and $q q q$ resonances exist because they are color singlets, so we should expect other color-singlet sectors of Hilbert space to possess resonances as well. The "four-quark" $q^{2} \bar{q}^{2}$ system was the subject of many detailed studies because it contains the first color-singlet multiquark system beyond three quarks, and because this system could couple to baryon-antibaryon systems through a single $q \bar{q}$ annihilation. Partly for this reason $q^{2} \bar{q}^{2}$ states were referred to as "baryonia". Although there were many reports of possible experimental baryonium states, and many detailed spectra were published in various models, no such states have yet been established. In sectors which support $q \bar{q}$ states the spectrum is already very complicated, so the issue of multiquark states remains somewhat obscure. However when one specializes to "smoking gun" systems such as the exotic $I=2$ channel, which is predicted to support a light $0^{++}$ $q^{2} \bar{q}^{2}$ level (at about $1.2 \mathrm{GeV}$ in the MIT bag model) but cannot have a $q \bar{q}$ state, there is no resonance in evidence [62].

The problems with the various theoretical models that led to erroneous predictions of discrete multiquark levels have been discussed by Isgur [63]. The novel feature of multiquark systems which the models missed is that, unlike $q \vec{q}$ and $q q q$, they need not exist as single color-singlet hadronic clusters; a $q^{2} \bar{q}^{2}$ system in general has some projection onto two colorsinglet $q \bar{q}$ mesons, and continuous deformation into two separate mesons appears to be energetically favored in most cases. This rearrangement into color singlets is called "fallapart" [64], and apparently excludes most single-hadron $q^{2} \bar{q}^{2}$ clusters as resonances. Fallapart would not be possible if the cluster had a mass lower than the threshold of the twohadron system it can rearrange into, which is why the question of the existence of multiquark clusters such as the $H u^{2} d^{2} s^{2}$ system is so interesting. The bag model predicts this state $81 \mathrm{MeV}$ below $\Lambda \Lambda$ threshold [64], but this prediction should be treated with caution because 
the bag model has previously given a misleading picture of multiquark states. The tentative evidence for dilambda hypernuclei [65] (if confirmed) makes the existence of an $H$ six-quark resonance well below $\Lambda \Lambda$ threshold appear very unlikely. Whether single multiquark clusters exist as resonances under any conditions is a detailed dynamical question, which should be investigated using models that allow the system itself freedom to choose between a single cluster or separate color singlets. At present it appears that single $q^{2} \bar{q}^{2}$ hadronic clusters may only exist as resonances in heavy-light systems such as $c^{2} \bar{q}^{2}$ [66].

It was the lack of sufficient freedom in the wavefunctions that led to the spurious prediction of many discrete baryonium levels; the models assumed that such states existed, and then gave predictions for the spectrum of these discrete levels. The first detailed study that allowed the $q^{2} \bar{q}^{2}$ system to choose between clusters and separate mesons as ground states was the variational study of the $0^{++}$sector by Weinstein and Isgur [67], which found that continuous deformation of a cluster into separate $q \bar{q}$ mesons was usually preferred energetically. The two exceptions found by Weinstein and Isgur will be discussed below.

\section{B. Experimental Molecule Candidates}

\section{1) Nuclei}

Lest one form the impression that hadronic molecules are controversial, note that the $\sim 10^{5}$ known nuclear levels are all hadronic molecules. Of course the term is usually applied to hadron pairs; even if we specialize to this restricted case, the deuteron can be cited as a noncontroversial example of a dominantly S-wave hadronic molecule. Its almost-bound $I=1, S=0$ partner is an example of another phenomenon which may appear elsewhere in the spectrum, a molecular resonance above threshold which is due to a strongly attractive final-state interaction. The existence of so many nuclear species is especially notable since the nucleon-nucleon system is rather unfavorable for the formation of bound states, due to the strong short-distance repulsive core. This suggests that many other families of bound hadrons may exist, although they may not be experimentally accessible except in special 
cases.

2) $f_{0}(975)$ and $a_{0}(980):$ The " $K \bar{K}$-molecules".

Weinstein and Isgur [67] found an exception to the fall-apart phenomenon in the scalar sector, with parameters corresponding to the $q s \bar{q} \bar{s}$ system. Here weakly-bound deuteronlike states of kaon and antikaon were found to be the ground states of the four-quark system; Weinstein and Isgur refer to these as "K $\overrightarrow{\mathrm{K}}$ molecules". The scalars $f_{0}(975)$ and $a_{0}(980)$ were obvious candidates for these states, having masses just below $K \bar{K}$ threshold and strong couplings to strange final states. Subsequently the $\gamma \gamma$ couplings of the $f_{0}(975)$ and $a_{0}(980)$ were found to be anomalously small relative to expectations for light ${ }^{3} \mathrm{P}_{0} q \bar{q}$ states $(q=u, d)$, as discussed in Refs. ( $[68,69])$. The status of the $K \bar{K}$ molecule assignment and the many points of evidence in its favor have been discussed recently by Weinstein and Isgur [70,71].

Although Morgan and Pennington have argued against a molecule interpretation of the $f_{0}(975)[72]$, their criticism applies to a $K \bar{K}$ potential model in which the $f_{0}(975)$ is a single pole in the scattering amplitude. The more recent work of Weinstein and Isgur $[70,71]$ incorporates couplings to open meson-meson channels and heavier ${ }^{3} \mathrm{P}_{0} q \bar{q}$ states, so the physical resonances are not only $|K \bar{K}\rangle$. Since there has been much criticism of the idea of a pure $K \bar{K}$ bound state, a direct quote from Weinstein and Isgur [70] (regarding the $I=0$ state) is appropriate:

"Despite its name and location, the " $K \bar{K}$ molecule" is not a simple $K \bar{K}$ bound state. Its stability is dependent on its couplings to the other $I=0$ channels and at $E=M_{S^{*}}$ the coupled-channel wavefunction has substantial components of the other states."

Although the $f_{0}$ and $a_{0}$ states remain dominantly $K \bar{K}$, these modifications may answer the objections of Morgan and Pennington. Pennington suggests that the term "deuteronlike" may be a misnomer, if couplings to other states than $K \bar{K}$ play an important rôle in these states [68]. Thus it appears that the important question regarding the $f_{0}$ and $a_{0}$ may be one 
of detail, specifically how large the subdominant non- $K \bar{K}$ components are in these states and how they can be observed experimentally.

3) $f_{1}(1420)$

Since the $f_{1}(1420)$ is above the $K^{*} K$ threshold of $1390 \mathrm{MeV}$ it is a candidate for a nonresonant threshold enhancement $\left(K^{*} \bar{K}+h . c.\right)$ rather than a molecular bound state. This possibility was suggested by Caldwell [73], and satisfies the criteria of lying just above the $K^{*} K$ threshold (antiparticle labels are implicit) and having quantum numbers allowed for that pair in S-wave. The apparent width of the enhancement should not be narrower than the intrinsic width of the $K^{*}$, and indeed the PDG values are similar, $\Gamma\left(f_{1}(1420)\right)=56 \pm 3$ $\mathrm{MeV}$ and $\Gamma\left(K^{*}\right)=50 \mathrm{MeV}$. Longacre [74] found that a model with an S-wave nonresonant $\left(K^{*} \bar{K}+h . c.\right)$ enhancement gives a good description of this state, and Isgur, Swanson and Weinstein [75] also favor this possibility. The (off-shell) $\gamma \gamma^{*}$ couplings of the $f_{1}(1420)$ relative to expectations for a $1^{++} s \bar{s}$ state may provide a test of the hadron-pair model.

Another test of this $K^{*} K$-assignment is in radiative transitions; the dominant radiative mode of a $K^{*} K$ system will arise from the radiative transition of the $K^{*}$ constituent, $K^{*} \rightarrow$ $K \gamma$, implying a partial width of

$$
\Gamma\left(f_{1}\left(K^{*} K\right) \rightarrow \gamma K \bar{K}\right) \approx 80 \mathrm{keV}
$$

and a characteristic pattern of preference for $K^{\circ} \bar{K}^{o}$ over $K^{+} K^{-}$by about a factor of two. An $s \bar{s}$ state would give a similar radiative partial width, $\Gamma\left(f_{1}(1420)(s \bar{s}) \rightarrow \gamma \phi\right) \approx 50 \mathrm{keV}$ if we scale from the $\Gamma\left(f_{2}^{\prime} \rightarrow \gamma \phi\right)=96 \mathrm{keV}$ of Godfrey and Isgur [3]. Although the radiative rates are similar, there is a crucial difference in the intermediate states: The $s \bar{s}$ decay is to $\gamma \phi$, so the final $K \bar{K}$ pair will originate from a $\phi(1019)$ peak. The $K \bar{K}$ events from a $K^{*} K$ system will instead peak at the invariant mass which results from the radiative decay $K^{*} \rightarrow K \gamma$ of an initial $K^{*} \bar{K}$ pair at rest, which is slightly above the $\phi$ mass. Due to a numerical coincidence these two masses are rather similar, so the most convincing tests would be the $\gamma K^{\circ} \bar{K}^{\circ}$ to $\gamma K^{+} K^{-}$ratio and the $\gamma K$ invariant mass distribution (obviously this will have a $K^{*}$ peak in 
the molecule assignment). Thus, the two $f_{1}(1420)$ assignments can be distinguished through a study of the charged and neutral final states and $\gamma K$ invariant mass distributions observed in $f_{1}(1420) \rightarrow \gamma K \bar{K}$.

4) $f_{0}(1710)$

Early references often considered this a glueball candidate, since it was discovered in a $\psi$ radiative decay [76] and has no obvious assignment in the $q \bar{q}$ spectrum. It was also discussed as a possible $q s \bar{q} \bar{s}$ multiquark state (a single cluster rather than a molecule), although the presence of a fall-apart coupling to $K \bar{K}$ makes this appear untenable. The $f_{0}(1710)$ appears unlikely to be a radially excited nonstrange ${ }^{3} \mathrm{P}_{0} q \bar{q}$ because it has a mass $110 \mathrm{MeV}$ below the Godfrey-Isgur prediction [3] and it has a very weak coupling to $\pi \pi$ final states, which makes both nonstrange $q \bar{q}$ and glueball assignments rather implausible (assuming naive flavor-singlet glueball couplings).

- The $f_{0}(1710)$ should also be considered a molecule candidate, of two vector mesons. There have been several suggestions in the literature regarding molecule assignment for this state, which differ primarily in the proposed binding mechanism; this leads to observable differences in the predicted decay modes.

Törnqvist [77] suggests a $K^{*} \bar{K}^{*}$ assignment for this state. If the $K^{*}$ s decay as free hadrons this leads one to expect a large partial width of $\Gamma\left(f_{0}(1710) \rightarrow K^{*} \bar{K}^{*}\right) \approx 2 \Gamma\left(K^{*}\right) \approx 100 \mathrm{MeV}$, making it the dominant decay mode, since the 1992 PDG gives the $f_{0}(1710)$ a total width of $146 \pm 12 \mathrm{MeV}$. This prediction appears to disagree with the branching fractions to other final states estimated by the PDG. Ericson and Karl [78] have studied the one-pion-exchange binding mechanism proposed by Törnqvist, and conclude that it may just provide sufficient attraction to bind $K^{*} \bar{K}^{*}$. They also conclude that this mechanism would predict several other vector-vector and meson-baryon molecules, such as $B^{*} B^{*}$ and $N \Sigma$.

Dooley et al. [79] assumed that a different mechanism was responsible for the dominant vector-vector interactions, specifically a OGE constituent interchange model which they had previously applied to several other channels (see previous section). This model predicts a 
very strong coupling between $K^{*} \bar{K}^{*}$ and $\omega \phi$ channels, found by Swanson [80] and represented using off-diagonal hadron-hadron potentials. Dooley et al. used these potentials in a multichannel generalization of the Weinstein-Isgur work, and found weakly bound molecules in several vector-vector systems. In the $I=0 q s \bar{q} \bar{s}$ sector they found a linear combination of two-meson basis states as a $0^{++}$ground state,

$$
\left|\Psi_{0}\left(q s \bar{q} \bar{s} 0^{++}\right)\right\rangle=\frac{1}{\sqrt{2}}\left(\left|K^{*} \bar{K}^{*}\right\rangle+|\omega \phi\rangle\right)
$$

and a $2^{++}$excited state somewhat higher in mass. This linear combination predicts $K \bar{K}$ and $\eta \eta$ branching fractions close to experimental values [81] and also gives reasonable results for the flavor-tagging $\psi \rightarrow V X$ hadronic decays, which suggest that the $f_{0}(1710)$ couples as if it were a strange-nonstrange mixed-flavor state. (A similar result is known for the $f_{0}(975)$ in these decays.) A $K^{*} \bar{K}^{*}$ decay mode is expected as in the pure $K^{*} \vec{K}^{*}$-molecule picture, but with a smaller branching fraction of about $35 \%$. The branching fraction of a $\left(K^{*} \bar{K}^{*}+\omega \phi\right) / \sqrt{2}$ molecule to $\pi \pi$ is more problematical; Dooley found it to be about $5 \%$ [81], consistent with experiment. A very characteristic electromagnetic decay mode in this assignment is $f_{0}(1710) \rightarrow \phi \pi^{0} \gamma$, with a branching fraction of $0.3 \%$, due to constituent$\omega$ radiative decay. It should also have an anomalously small $\gamma \gamma$ coupling relative to a nonstrange $I=0 q \vec{q}$ state such as the $f_{2}(1274)[82]$.

A search for a $K^{*} \bar{K}^{*}$ mode is clearly the most important test of the vector-molecule models of the $f_{0}(1710)$, since both models expect a large $K^{*} \bar{K}^{*}$ branching fraction. Of course one $K \pi$ combination will be skewed downwards from the free $K^{*}$ mass by slightly more than $E_{B}$, assuming that this acts as a sequential decay, $f_{0}(1710) \rightarrow K^{*}(\bar{K} \pi)$ followed by decay of the free recoiling $K^{*}$.

5) $\Lambda(1405)$

In meson-baryon sectors, the $\frac{1}{2}^{-} \Lambda(1405)$ has long been considered a candidate $\bar{K} N$ bound state [83], since it is just below the $\bar{K} N$ threshold, has $\mathrm{S}$-wave $\bar{K} N$ quantum numbers, and is nearly $150 \mathrm{MeV}$ below the mass expected for the lightest $\frac{1}{2}^{-} \Lambda^{*}$ baryon in the quark model 
[4]. (The decay amplitudes however are consistent with a $u d s$ assignment [4].) Theoretical study of this channel has been incomplete, however, because of the complications of open channels and $q \bar{q}$ annihilation; a model which includes mixing in the full $N \bar{K}-\Sigma \pi-u d s$ system is required for a complete study of this state. The radiative partial widths to $\gamma \Lambda$ and $\gamma \Sigma^{0}$ compared to quark model predictions for a $u d s$ baryon may allow a convincing test of the molecule assignment. A wide range of theoretical numbers has been reported for these radiative widths [84], so one should be careful to use techniques which give reliable results for well-established $q q q$ quark model states.

6) $Z^{*} s$ and dibaryons

We conclude this section with a reminder that there may be hadronic molecules in other sectors of Hilbert space, which have received little recent attention because they do not correspond to $q \bar{q}$ or $q q q$ flavor states in the quark model or because of the lack of appropriate experimental facilities.

One especially interesting system, which should soon be accessible to experiments at DA $\Phi N E$ and perhaps CEBAF, is the kaon-nucleon system. Possible resonances in the $q^{4} \vec{s}$ sector are known as $Z^{*}$ s, and although there have been indications of such states for many years in elastic KN scattering [85], the lack of clear evidence for multiquark states and the uncertainties of partial wave analyses in the $K N$ system have left the possibility of such states a controversial question. In our recent theoretical study of $K N, K^{*} N, K \Delta$ and $K^{*} \Delta$ systems [86] we found that several of these channels, notably the minimum total spin, minimum total isospin ones, have strongly attractive interactions. The experimental reports of $Z^{*}$ resonances may represent observations of final state enhancements or even of mesonbaryon bound states just below threshold. Clarification of this issue will require accurate partial wave analyses of $K N$ scattering, and data on the inelastic channels $K N \rightarrow K^{*} N, K \Delta$ and $K^{*} \Delta$ would also be very valuable for the study of possible $Z^{*}$ states. Here the theoretical calculations should be more reliable since these reactions are annihilation-free at valence quark level. 
Finally, there are controversial reports of resonances in partial wave analyses of elastic NN scattering [87], and these "dibaryon" resonances may also include S-wave baryon-baryon molecule states. This system too is relatively straightforward theoretically because it is annihilation-free, and a few $\Delta \Delta$ channels (notably $I=0, S=1, I=1, S=0 ; I=0, S=3$ and $I=3, S=0$ ) have been cited by theorists as the most likely for the formation of nonstrange baryon-baryon resonances $[88,89]$.

7) Other possible molecules.

There are many other possible molecular states, which can only be mentioned briefly here. In the meson sector these include the $f_{0}(1500)$ glueball candidate, which might be a nonstrange vector-vector system [77,90]. This would explain the weakness of the $K \bar{K}$ mode. The $f_{0}(1365)$ should also be considered a possible vector-vector molecule, in view of its very large coupling to $\rho \rho$ despite the near absence of phase space. The $2^{++}$state reported by VES [57] in $\rho^{\circ} \rho^{\circ}$ is another possible vector-vector molecule, although its appearance in the $\rho \rho \mathrm{D}$-wave may be a problem. The $\psi(4040)$, which shows a strong preference for $D \bar{D}$ over $D^{*} \bar{D}^{*}$ (opposite to expectations from phase space) was one of the earliest molecule candidates [91]. As the $c \bar{c}$ assignment for this state is a $3 \mathrm{~S}$ radial excitation, this anomalous branching fraction may be due to a node in the decay amplitude near the $D^{*} \bar{D}^{*}$ momentum [92].

\section{Signatures for Molecules}

Leaving aside questions of detailed dynamics, there are several obvious signatures for hadron-pair molecules that may be abstracted from the more convincing molecule candidates. These signatures are:

1) $J^{P C}$ and flavor quantum numbers of an $L=0$ hadron pair.

The residual "nuclear" strong forces that bind molecules are of such short range that $L>0$ 
molecules appear unlikely in light hadronic systems. Actually there is a possible exception, the $\psi(4040)$, which couples so strongly to $D^{*} \bar{D}^{*}$ that it was suggested as a P-wave $D^{*} \bar{D}^{*}$ molecule (note $E_{B} \approx 0$ ) some time ago [91]. This exception may be possible because heavier mesons bind more easily, and the light quarks insure relatively strong interactions between them. Should this state actually be a P-wave charmed-meson molecule, a spectrum of more deeply-bound S-wave charm molecules is anticipated [93].

2) A binding energy of at most about $50-100 \mathrm{MeV}$.

From the uncertainty principle; a minimum separation of $\approx 1 \mathrm{fm}$ is required for hadrons to maintain separate identities, which gives $E_{B} \sim 1 /\left(2 \mu R^{2}\right) \sim 1 /(1 \mathrm{GeV}) \cdot(1 \mathrm{fm})^{2} \approx 50 \mathrm{MeV}$, and of course a factor-of-two uncertainty is plausible in this simple estimate. For comparison, Weinstein and Isgur find an rms $K \bar{K}$ separation of about $1.7 \mathrm{fm}$ in their model of the $f_{0}(975)$ and $a_{0}(980)$, which have $E_{B} \approx 10-20 \mathrm{MeV}$. Note also that an attractive interaction may lead to a final-state enhancement in S-wave just above threshold, which may or may not be resonant; the $I=1, S=0$ partner of the deuteron is an example of such a resonance, and the $f_{1}(1420)$ may be an example of a nonresonant final-state enhancement.

3) Strong couplings to constituent channels.

As an example, the anomalously large coupling of the $f_{0}(975)$ to $K \bar{K}$, as indicated by $B(K \bar{K}) / B(\pi \pi) \approx 1 / 4$ despite the near absence of $K \bar{K}$ phase space, is an important clue that it is not a nonstrange $q \bar{q}$ state.

4) Anomalous EM couplings relative to expectations for conventional quark model states.

The $f_{0}(975)$ for example has a tiny $\gamma \gamma$ partial width of perhaps $0.2 \mathrm{Kev}$ to $0.6 \mathrm{Kev}$ (depending on the analysis) [68]. This small $\gamma \gamma$ width is expected for a $K \bar{K}$ molecule [69], but for a nonstrange ${ }^{3} \mathrm{P}_{0} q \bar{q}$ state the quark model predicts about $3 \mathrm{Kev}$ [94], as has been found for the broad $f_{0}(\approx 1300)[95]$. Close, Isgur and Kumano [96] suggest a related test for the $f_{0}(975)$ 
and $a_{0}(980)$ involving the radiative decays $\phi \rightarrow \gamma\left(f_{0}, a_{0}\right)$, which may be possible at DA $\Phi$ NE and CEBAF [97].

\section{The Importance of Scattering Amplitudes}

Since molecular bound states are a special aspect of $2 \rightarrow 2$ hadron scattering amplitudes, one might anticipate that an understanding of these scattering amplitudes will lead to reliable predictions of molecules. Theoretical work along these lines is in progress; at present there are different predictions for molecules depending on the scattering mechanism assumed.

In one pion exchange models $[77,78]$ many bound states are predicted which should be experimentally observable. Here I would like to discuss scattering calculations in terms of quark-gluon forces (see $[79,80,88,86,98,99]$ and references cited therein).

Hadron-hadron scattering amplitudes at low energies are generally thought to involve the quark-gluon interaction nonperturbatively, so although calculations of meson-meson and baryon-baryon interactions at the quark-gluon level have been rather successful, they have typically used complicated nonperturbative methods such as resonating group or variational techniques. Extension of this work to channels such as vector-vector has been slow largely because of the difficulty of applying these methods, although some variational and Monte Carlo results have been reported for special cases, including an extension of the WeinsteinIsgur work to $I=2 \rho \rho[100]$.

Recently our collaboration has found evidence that the Ps-Ps scattering amplitudes found by Weinstein and Isgur (in channels without $q \bar{q}$ annihilation) are actually dominated by perturbative diagrams, although "higher-twist" contributions in the form of external $q \bar{q}$ wavefunctions attached to the diagrams are an essential, nonperturbative aspect of the scattering amplitudes [80,98]. We initially studied $I=2 \pi \pi$ [98] and $I=3 / 2 K \pi$ [99] and found that OGE followed by constituent interchange dominates these scattering amplitudes, and leads to results which are numerically very similar to the Ps-Ps potentials found variationally by Weinstein and Isgur. Recent lattice QCD results for the $I=2 \pi \pi$ scattering length support 
our conclusion regarding the dominance of these diagrams [101]. We refer to these perturbative diagrams with external wavefunctions attached as "quark Born diagrams". With SHO quark model wavefunctions these lead to overlap integrals that can often be evaluated in closed form, and the results for $\pi \pi$ and $K \pi$ S-wave phase shifts are in excellent agreement with experiment over the entire range of energies studied given standard quark model parameters. We have similarly found good agreement in the $I=0$ and $I=1 \mathrm{KN}$ system [86] (albeit with some problems at higher energies which may be due to the assumption of single-Gaussian nucleon wavefunctions). In our study of $N N, N \Delta$ and $\Delta \Delta$ [88] we found the strongest diagonal attraction in the $I=0, S=1 \Delta \Delta$ channel, in agreement with the variational work of Maltman [89]. Very recently we have studied the $N_{s}=2$ baryon-baryon channel [102], and we agree with Oka, Shimizu and Yazaki and Straub et al. [103] that quark model forces lead to a repulsive $\Lambda \Lambda$ core interaction; of the six $N_{s}=2$ octet-octet channels we find that only $I=0, S=0 \Sigma \Sigma$ has an attractive core. It is reassuring that exactly the same conclusion was reached by Oka et al. [103] using nonperturbative resonating group methods.

Given this reasonably successful description of hadron-hadron scattering at low energies, can we proceed to study all experimentally accessible channels and see in which we expect hadronic molecules to form? Unfortunately this is not yet possible. The difficulty is that $q \bar{q}$ annihilation appears to be the dominant effect when allowed (as in $I=0,1 \pi \pi$ and $I=1 / 2$ $K \pi$ ), so a realistic description of scattering amplitudes in these channels requires accurate modelling of the couplings of different sectors of Hilbert space (as in $\pi \pi \rightarrow f_{0}(q \bar{q}) \rightarrow \pi \pi$ ). On closer examination of the Weinstein-Isgur results [71] it now appears that both level repulsion against higher-mass s-channel $q \tilde{q}$ resonances and nonresonant scattering are needed for the simultaneous binding of $I=0$ and $I=1 K \bar{K}$ systems. (Without level repulsion against the $q \bar{q}^{3} \mathrm{P}_{0}$ states at $\approx 1.3 \mathrm{GeV}$ only the $I=0$ molecule binds.) The existence of molecules in some channels may be due entirely to level repulsion against a more massive quark model state, and the hadronic couplings of most quark-model states are not well enough established to model this level repulsion accurately. Remarkably, the $q \bar{q}$ pair production process is 
still rather poorly understood (for a recent study see [104]), and is usually treated using phenomenological models such as ${ }^{3} \mathrm{P}_{0}$ that have no clear relation to QCD. Accurate modelling of hadronic forces in channels with annihilation, and hence reliable predictions of molecules, must await a better understanding of the $q \bar{q}$ annihilation mechanism.

\section{ACKNOWLEDGEMENTS}

It is a pleasure to thank D.Bugg for his kind invitation to present these lectures to the NATO Summer School on the Confinement Problem, held at Queen Mary and Westfield College and at the University of Swansea. 'I would also like to thank my colleagues and collaborators, notably E.S.Ackleh, C.Amsler, J.Beene, S.Capstick, S.U.Chung, F.E.Close, G.Condo, K.Danyo, A.Dzierba, P.Geiger, S.Godfrey, N.Isgur, P.R.Page and E.S.Swanson for their assistance in preparation of these notes. I sincerely hope that the attendees of the Summer School will find an exciting and rewarding career in the study of QCD and hadron physics.

This research was sponsored in part by the United States Department of Energy under contract DE-AC05-840R21400, managed by Lockheed Martin Energy Systems, Inc.

\section{DISCLAIMER}

This report was prepared as an account of work sponsored by an agency of the United States Government. Neither the United States Government nor any agency thereof, nor any of their employees, makes any warranty, express or implied, or assumes any legal liability or responsibility for the accuracy, completeness, or usefulness of any information, apparatus, product, or process disclosed, or represents that its use would not infringe privately owned rights. Reference herein to any specific commercial product, process, or service by trade name, trademark, manufacturer, or otherwise does not necessarily constitute or imply its endorsement, recommendation, or favoring by the United States Government or any agency thereof. The views and opinions of authors expressed herein do not necessarily state or reflect those of the United States Government or any agency thereof. 
[1] W.Marciano and H.Pagels, Phys. Rep. 36C, 137-276 (1978).

[2] Particle Data Group, Phys. Rev. D50, 1177 (1994).

[3] S.Godfrey and N.Isgur, Phys. Rev. D32, 189 (1985).

[4] S.Capstick and N.Isgur, Phys. Rev. D34, 2809 (1986).

[5] P.Geiger and N.Isgur, Phys. Rev. D47, 5050 (1993); Phys. Rev. Lett. 67, 1066 (1991); Phys. Rev. D44, 799 (1991); ibid., D41, 1595 (1990).

[6] E.Eichten, K.Gottfried, T.Kinoshita, K.D.Lane and T.M.Yan, Phys. Rev. D17, 3090 (1978); Phys. Rev. D23, 203 (1980).

[7] T.A.Armstrong et al., E760 Collaboration, Phys. Rev. Lett. 69, 2337 (1992).

[8] S.Godfrey, Phys. Rev. D31, 2375 (1985).

[9] The $a_{0}(1450)$ is reviewed by S.Spanier, contribution to HADRON95.

[10] A.LeYaouanc, L.Oliver, O.Pene and J.Raynal, Phys. Rev. D8, 2223 (1973); ibid., D9, 1415 (1974); ibid., D11, 1272 (1975); see also L.Micu, Nucl. Phys. B10, 521 (1969).

[11] E.S.Ackleh, T.Barnes and E.S.Swanson, Oak Ridge National Laboratory report ORNL-CTP95-09.

[12] E.S.Ackleh and T.Barnes, Phys. Rev. D45, 232 (1992); T.Barnes, in Proceedings of the Ninth International Workshop on Photon-Photon Collisions (La Jolla, 22-26 March 1992), eds. D.O.Caldwell and H.P.Paar (World Scientific, 1992), pp.263-282.

[13] D. Antreasyan et al., Z. Phys. C48, 561 (1990); H.J. Behrend et al., Z. Phys. C46, 583 (1990); K.-H. Karch et al., Z. Phys. C54, 33 (1992).

[14] F.E.Close, Rep. Prog. Phys. 51, 833 (1988). 
[15] For the earlier LGT glueball literature see for example C.Michael and M.Teper, Nucl. Phys. B314, 347 (1989); P.deForcrand, G.Schierholz, H.Schneider and M.Teper, Phys. Lett. B152, 107 (1985); G.Berg, Nucl. Phys. B221, 109 (1983); and references cited therein.

[16] G.Bali et al., Phys. Lett. B309, 378 (1993).

[17] H.Chen et al., IBM report IBM-HET-94-1 (contribution of A. Vaccarino to Lattice 93).

[18] J. Sexton et al., IBM report IBM-HET-94-5 (contribution to Lattice 94).

[19] C.Amsler and F.E.Close, "Evidence for Glueballs", Rutherford Laboratory and CERN report CCL-TR-95-003 (April 1995); ibid., Phys. Lett. B353, 385 (1995) and Rutherford Laboratory report RAL-TR-95-003 (July 1995).

[20] V.V.Anisovich et al., Phys. Lett. B323, 233 (1994); C.Amsler, in Proceedings of the XXVII Int. Conf. on High Energy Physics (Glasgow, 20-27 July 1994), Zürich report UZH-PH-50/94.

[21] C.Amsler, personal communication.

.[22] D.Bugg et al., Phys. Lett. B353, 378 (1995).

[23] T.Huang, contribution to the Argonne Workshop on a Tau-Charm Factory (June 1995); see also T.Huang et al., CCAST report BIHEP-TH-95-11.

[24] S.Godfrey, R.Kokoski and N.Isgur, Phys. Lett. B141, 439 (1984).

[25] S.Godfrey, personal communication; H.G.Blundell and S.Godfrey, "The $\xi(2220)$ Revisited: Strong Decays of the $1^{3} F_{2}$ and $1^{3} F_{4} s \bar{s}$ Mesons.", Carleton University report OCIP/C 95-11.

[26] T.Barnes, Caltech Ph.D. thesis (1977), unpublished; T.Barnes, Nucl. Phys. B158, 171 (1979); T.Barnes and F.E.Close, Phys. Lett. 116B, 365 (1982); M.Chanowitz and S.R.Sharpe, Nucl. Phys. B222, 211 (1983); T.Barnes, F.E.Close and F.deViron, Nucl. Phys. B224, 241 (1983); M.Flensburg, C.Peterson and L.Sköld, Z. Phys. C22, 293 (1984).

[27] D.Horn and J.Mandula, Phys. Rev. D17, 898 (1978). 
[28] T.Barnes, "The Bag Model and Hybrid Mesons", in Proceedings of the SIN Spring School on Strong Interactions (Zuoz, Switzerland, April 9-17, 1985). Also distributed as University of Toronto report UTPT-85-21 (April 1985).

·29] M.Tanimoto, Phys. Lett. 116B, 198 (1982); Phys. Rev. D27, 2648 (1983); A.LeYaouanc, L.Oliver, O.Pène, J.-C.Raynal and S.Ono, Z. Phys. C28, 309 (1985); F.Iddir, A.LeYaouanc, L.Oliver, O.Pène, J.-C.Raynal and S.Ono, Phys. Lett. B205, 564 (1988); S.Ishida, H.Sawazaki, M.Oda and K.Yamada, Phys. Rev. D47, 179 (1992); Prog. Theor. Phys. 82, 119 (1989).

[30] J.M.Cornwall and S.F.Tuan, Phys. Lett. B136, 110 (1984).

[31] N.Isgur and J.Paton, Phys. Lett. 124B, 247 (1983).

[32] J.Merlin and J.Paton, J. Phys. G11, 439 (1985).

[33] N.Isgur and J.Paton, Phys. Rev. D31, 2910 (1985).

[34] J.Merlin and J.Paton, Phys. Rev. D35, 1668 (1987).

[35] J.Merlin, Oxford University Ph.D. thesis (unpublished); J.Paton, personal communication.

[36] N.Isgur, R.Kokoski and J.Paton, Phys. Rev. Lett. 54, 869 (1985).

[37] T.Barnes, F.E.Close and E.S.Swanson, Oak Ridge National Laboratory / Rutherford Laboratory Report ORNL-CTP-95-02 / RAL-94-106, hep-ph/9501405, Phys. Rev. D (to appear).

[38] F.E.Close and P.R.Page, Nucl. Phys. B443, 233 (1995).

[39] F.E.Close and P.R.Page, Rutherford Laboratory report RAL-94-122, hep-ph/9412301.

[40] P.Hasenfratz, R.R.Horgan, J.Kuti and J.-M.Richard, Phys. Lett. 95B, 299 (1980).

[41] S.Perantonis and C.Michael, Nucl. Phys. B347, 854 (1990), and references cited therein.

[42] J.I.Latorre, S.Narison, P.Pascual and R.Tarrach, Phys. Lett. 147B, 169 (1984); J.I.Latorre, P.Pascual and S.Narison, Z. Phys. C34, 347 (1987); S.Narison, "QCD Spectral Sum Rules", Lecture Notes in Physics Vol.26, p.375 (World Scientific, 1989). 
[43] J.Govaerts, F.deViron, D.Gusbin and J.Weyers, Phys. Lett. 128B, 262 (1983); (E) Phys. Lett. 136B, 445 (1983); J.Govaerts, L.J.Reinders, H.R.Rubinstein and J.Weyers, Nucl. Phys. B258, 215 (1985); J.Govaerts, L.J.Reinders and J.Weyers, Nucl. Phys. B262, 575 (1985); J.Govaerts, L.J.Reinders, P.Francken, X.Gonze and J.Weyers, Nucl. Phys. B284, 674 (1987).

[44] J.Govaerts, F.deViron, D.Gusbin and J.Weyers, Nucl. Phys. B248, 1 (1984).

[45] See for example T.Barnes, ORNL-CCIP-93-11 / RAL-93-065 and F.E.Close, RAL-93-053, in Proceedings of the Third Workshop on the Tau-Charm Factory (Marbella, Spain, 1-6 June 1993); T.Barnes, ORNL-CCIP-93-14 / RAL-93-069 in Proceedings of the Conference on Exclusive Reactions at High Momentum Transfers (Marciana Marina, Elba, Italy, 2426 June 1993); F.E.Close, Rep. Prog. Phys. 51, 833 (1988); C.Dover, in Proceedings of the Second Biennial Conference on Low Energy Antiproton Physics (Courmayeur, 14-19 Sept. 1992); A.Dzierba, Indiana University report IUHEE-93-2, in Proceedings of the BNL meeting on Future Directions in Particle and Nuclear Physics at Multi-GeV Hadron Facilities (Brookhaven, N.Y. 4-6 March 1993); S.Godfrey, in Proceedings of the BNL Workshop on Glueballs, Hybrids and Exotic Hadrons (AIP, 1989), ed. S.-U. Chung; D.Hertzog, Nucl. Phys. A558, 499c (1993); N.Isgur, CEBAF-TH-92-31, in Proceedings of the XXVI International Conference on High Energy Physics (Dallas, August 1992); G.Karl, Nucl. Phys. A558, 113c (1993).

[46] G.Condo et al., Phys. Rev. D43, 2787 (1991); the same state may have been seen earlier by D.Aston et al., Nucl. Phys. B189, 15 (1981).

[47] J.H.Lee et al., Phys. Lett. B323, 227 (1994).

[48] F.E.Close and P.R.Page, Oxford University / Rutherford Laboratory report OUTP-95-13P / RAL-95-122, hep-ph/9507407.

[49] T.Barnes, in Proceedings of the 3rd Workshop on the Tau Charm Factory (Edition Frontieres 1994), eds. J.Kirkby and R.Kirkby, p.41. 
[50] F.E.Close, in Proceedings of the 3rd Workshop on the Tau Charm Factory (Edition Frontieres 1994), eds. J.Kirkby and R.Kirkby, p.73.

[51] D.Alde et al., Phys. Lett. B205, 397 (1988).

[52] H.Aoyagi et al., Phys. Lett. B314, 246 (1993).

[53] G.M.Beladidze et al., Phys. Lett. B313, 276 (1993).

[54] S.U.Chung, personal communication.

[55] A.Dzierba, personal communication.

[56] M.Doser, personal communication; see also J.LoSecco, E852 Collaboration, in preparation.

[57] D.Rybachikov, contribution to HADRON95.

[58] A.Donnachie and Yu. Kalashnikova, Z.Phys C59, 621 (1993); A.Donnachie, Yu. Kalashnikova and A.Clegg, Manchester University Report C-TH-93/10.

[59] A.B.Clegg and A.Donnachie, Z. Phys. C62, 455 (1994).

[60] G.Busetto and L.Oliver, Z. Phys.C 20, 247 (1983).

[61] R.Kokoski and N.Isgur, Phys. Rev. D35, 907 (1987).

[62] W.Hoogland et al., Nucl. Phys. B126, 109 (1977).

[63] N.Isgur, Acta Physica Austraica, Suppl. XXVII, 177 (1985).

[64] R.L.Jaffe, Phys. Rev. Lett. 38, 195, 617E (1977).

[65] S.Aoki et al., Prog. Theor. Phys. 85, 1287 (1991), and references cited therein.

[66] J.P.Ader, J.M.Richard and P.Taxil, Phys. Rev. D25, 2370 (1982); G.Grondin, unpublished.

[67] J.Weinstein and N.Isgur, Phys. Rev. Lett. 48, 659 (1982); Phys. Rev. D27, 588 (1983); see also A.Astier et al., Phys. Lett. B25, 294 (1967); A.B.Wicklund et al., Phys. Rev. Lett. 45, 1469 (1980). 
[68] M.R.Pennington, University of Durham report DTP-94/26 (April 1994), to appear in Proceedings of the Meeting on Two-Photon Physics from DAФNE to LEP200 and Beyond, eds. F.Kapusta and J.Parisi (Paris, February 1994).

[69] T.Barnes, Phys. Lett. 165B, 434 (1985); E.P.Shabalin, Yad. Fiz. 46, 852 (1987); T.N.Truong, in Proceedings of the HADRON '89 International Meeting on Hadron Spectroscopy (Ajaccio, 1989), pp.645; N.Brown and F.E.Close, Rutherford Laboratory report RAL-91-085.

[70] J.Weinstein and N.Isgur, Phys. Rev. D41, 2236 (1990).

[71] J.Weinstein, Phys. Rev. D47, 911 (1993).

[72] K.L.Au, D.Morgan and M.R.Pennington, Phys. Rev. D35, 1633 (1987); D.Morgan and M.R.Pennington, Phys. Lett. 258B, 444 (1991); ibid., Rutherford Laboratory report RAL-92070 (December 1992).

[73] D.O.Caldwell, Mod. Phys. Lett. A2, 771 (1987); Proceedings of the BNL Workshop on Glueballs, Hybrids and Exotic Mesons (Upton, N.Y.29 August - 1 September 1988), ed. S.U.Chung (AIP 1989), pp.465-471.

[74] R.S.Longacre, Phys. Rev. D42, 874 (1990).

[75] N.Isgur, E.S.Swanson and J.Weinstein, work in progress.

[76] C.Edwards et al., Phys. Rev. Lett. 48, 458 (1982).

[77] N.Törnqvist, in Proceedings of the International Conference on Hadron Spectroscopy "HADRON '91", (World Scientific, 1992; eds. S.Oneda and D.C.Peaslee), pp.795-798; Phys. Rev. Lett. 67, 556 (1991).

[78] T.E.O.Ericson and G.Karl, Phys. Lett. B309, 426 (1993); G.Karl, Nucl. Phys. A558, 113c (1993).

[79] K.Dooley, E.S.Swanson, and T.Barnes, Phys. Lett. 275B, 478 (1992); K.Dooley, in Proceedings of the 4th International Conference on Hadron Spectroscopy "Hadron '91" (College Park, 
Md. 12-16 August 1991), (World Scientific, 1992), pp.789-794.

[80] E.S.Swanson, Ann. Phys. (NY) 220, 73 (1992).

·[81] K.Dooley, Ph.D. thesis, University of Toronto (1993).

[82] E.S.Ackleh, K.Dooley and Z.P.Li, University of Toronto report UTPT-92-03.

[83] R.H.Dalitz and S.F.Tuan, Ann. Phys. (NY) 3, 307 (1960); J.J.Sakurai, Ann. Phys. (NY) 11, $1(1960)$.

[84] H.Burkhardt and J.Lowe, Phys. Rev. C44, 607 (1991).

[85] J.S.Hyslop, R.A.Arndt, L.D.Roper and R.L.Workman, Phys. Rev. D46, 961 (1992).

[86] T.Barnes and E.S.Swanson, Phys. Rev. C49, 1166 (1994); T.Barnes, in Proceedings of the DAPHNE95 Workshop on Physics and Detectors for the Frascati $\Phi$ Factory (4-7 April 1995, Frascati, Italy). See also K.Maltman and S.Godfrey, Nucl. Phys. A452, 669 (1986), who reach rather different conclusions regarding attractive channels.

[87] R.A.Arndt, L.D.Roper, R.L.Workman and M.W.McNaughton, Phys. Rev. D45, 3995 (1992).

[88] T.Barnes, S.Capstick, M.D.Kovarik and E.S.Swanson, Phys. Rev. C48, 539 (1993).

·[89] K.Maltman, Nucl. Phys. A438, 669 (1985);see also T.Goldman et al., Phys. Rev. C39, 1889 (1989); M.Cvetic et al., Phys. Lett. 93B, 489 (1980); T.Kamae and T.Fujita, Phys. Rev. Lett. 38,471 (1977).

[90] Yu.S.Kalashnikova, in Proceedings of the International Conference on Hadron Spectroscopy “HADRON '91", (World Scientific, 1992; eds. S.Oneda and D.C.Peaslee), pp.777-782.

[91] V.A.Novikov et al., Phys. Rep. C41, 1 (1978); M.B.Voloshin and L.B.Okun, JETP Lett. 23, 333 (1976).

[92] P.R.Page, Nucl. Phys. B446, 189 (1995); see also A.LeYaouanc et al., Phys. Lett. B71, 397 (1977); ibid., Phys. Lett. B72, 57 (1977). 
[93] A.DeRújula, H.Georgi and S.L.Glashow, Phys. Rev. Lett. 38, 317 (1977); S.Iwao, Lett. Nuovo Cimento 28, 305 (1980).

[94] Z.P.Li, F.E.Close and T.Barnes, Phys. Rev. D43, 216 (1991); E.S.Ackleh, University of Tennessee Ph.D. thesis (1993); T.Barnes, in Proceedings of the Ninth International Workshop on Photon-Photon Collisions (La Jolla, 22-26 March 1992), eds. D.O.Caldwell and H.P.Paar (World Scientific, 1992), pp.263-282; S:Gerasimov, unpublished.

[95] J.K.Bienlein (Crystal Ball Collaboration), in Proceedings of the Ninth International Workshop on Photon-Photon Collisions (La Jolla, 22-26 March 1992), eds. D.O.Caldwell and H.P.Paar (World Scientific, 1992), pp.241-257.

[96] F.E.Close, N.Isgur and S.Kumano, Nucl. Phys. B389, 513 (1993).

[97] A.Dzierba et al., CEBAF proposal E-94-016; A.Dzierba, "Measuring Rare Radiative Decays of the $\Phi$ Meson at CEBAF", in Proceedings of the Second Workshop on Physics and Detectors for DAPHNE (DAPHNE95).

[98] T.Barnes and E.S.Swanson, Phys. Rev. D46, 131 (1992); for closely related work on mesonmeson scattering see B.Masud, J.Paton, A.M.Green and G.Q.Liu, Nucl. Phys. A528, 477 (1991); B.Masud, "A Model for $q^{2} \bar{q}^{2}$ Systems, Illustrated by an Application to $K \bar{K}$ Scattering", University of Helsinki report HU-TFT-93-30 (November 1993); D.Blaschke and G.Röpke, Phys. Lett. B299, 332 (1993); A.LeYaouanc, L.Oliver, O.Péne and J.-C.Raynal, Phys. Rev. D42, 3123 (1990).

[99] T.Barnes, E.S.Swanson and J.Weinstein, Phys. Rev. D46, 4868 (1992).

[100] G.Grondin, E.S.Swanson and J.Weinstein, unpublished.

[101] S.R.Sharpe, personal communication; R.Gupta, A.Patel and S.R.Sharpe, Nucl. Phys. B383, 309 (1992).

[102] T.Barnes and J.Weinstein, in preparation. 
[103] M.Oka, K.Shimizu and K.Yazaki, Nucl. Phys. A464, 700 (1987). U.Straub, Z.-Y.Zhang, K.Bräuer, A.Fä $\beta$ ler and S.B.Khadkikar, Phys. Lett. 200B, 241 (1988).

[104] P.Geiger and E.S.Swanson, "Distinguishing Among Strong Decay Models", MIT report MITCTP-2295 (April 1994).

[105] C.Michael, in Proceedings of the Workshop on QCD: 20 Years Later (Aachen, 9-13 June 1992).

[106] C.Amsler et al., Phys. Lett. B322, 431 (1994). 


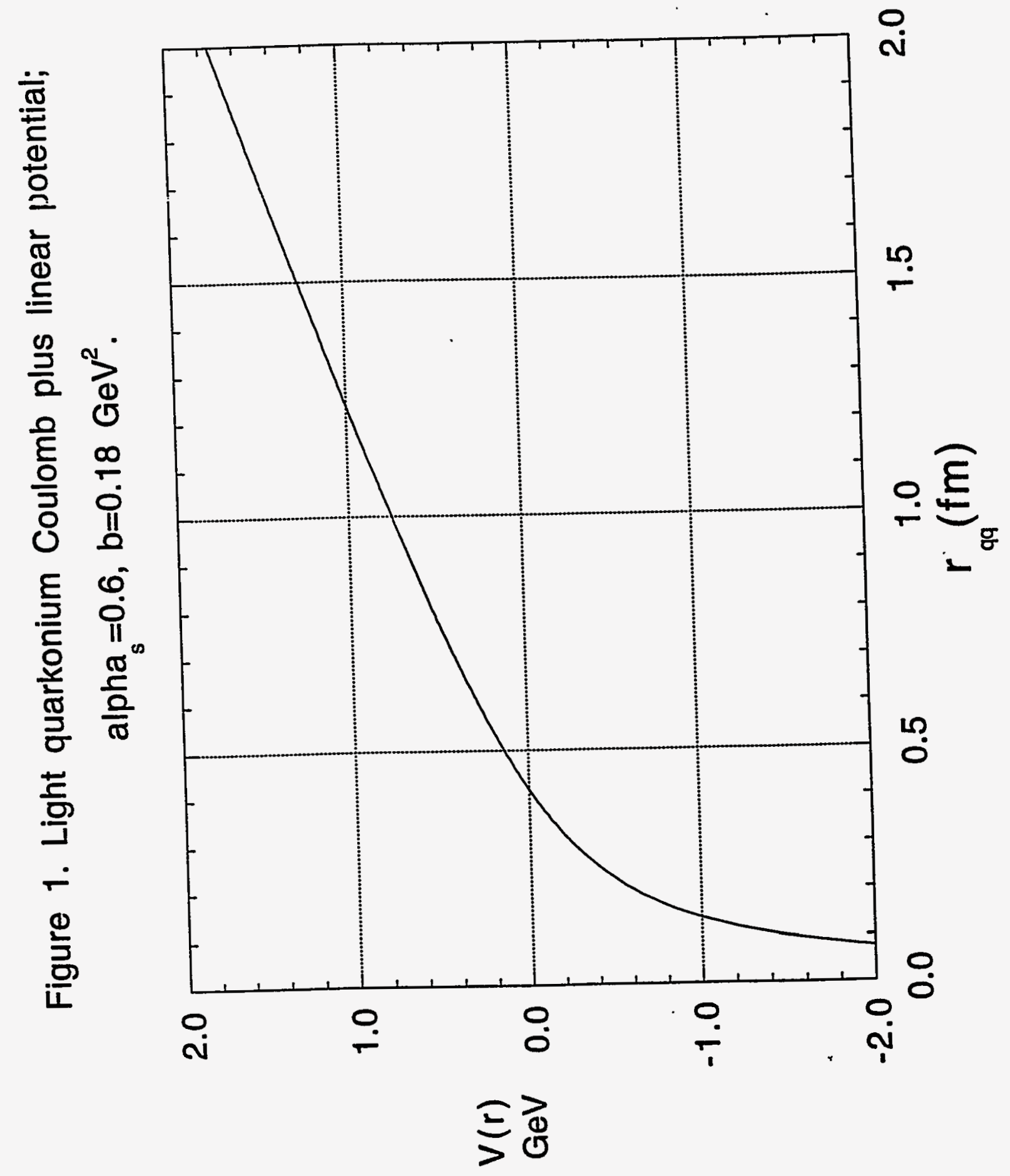


Figure 2. The charmonium spectrum; experiment (lines) versus a relativized potential model (points).

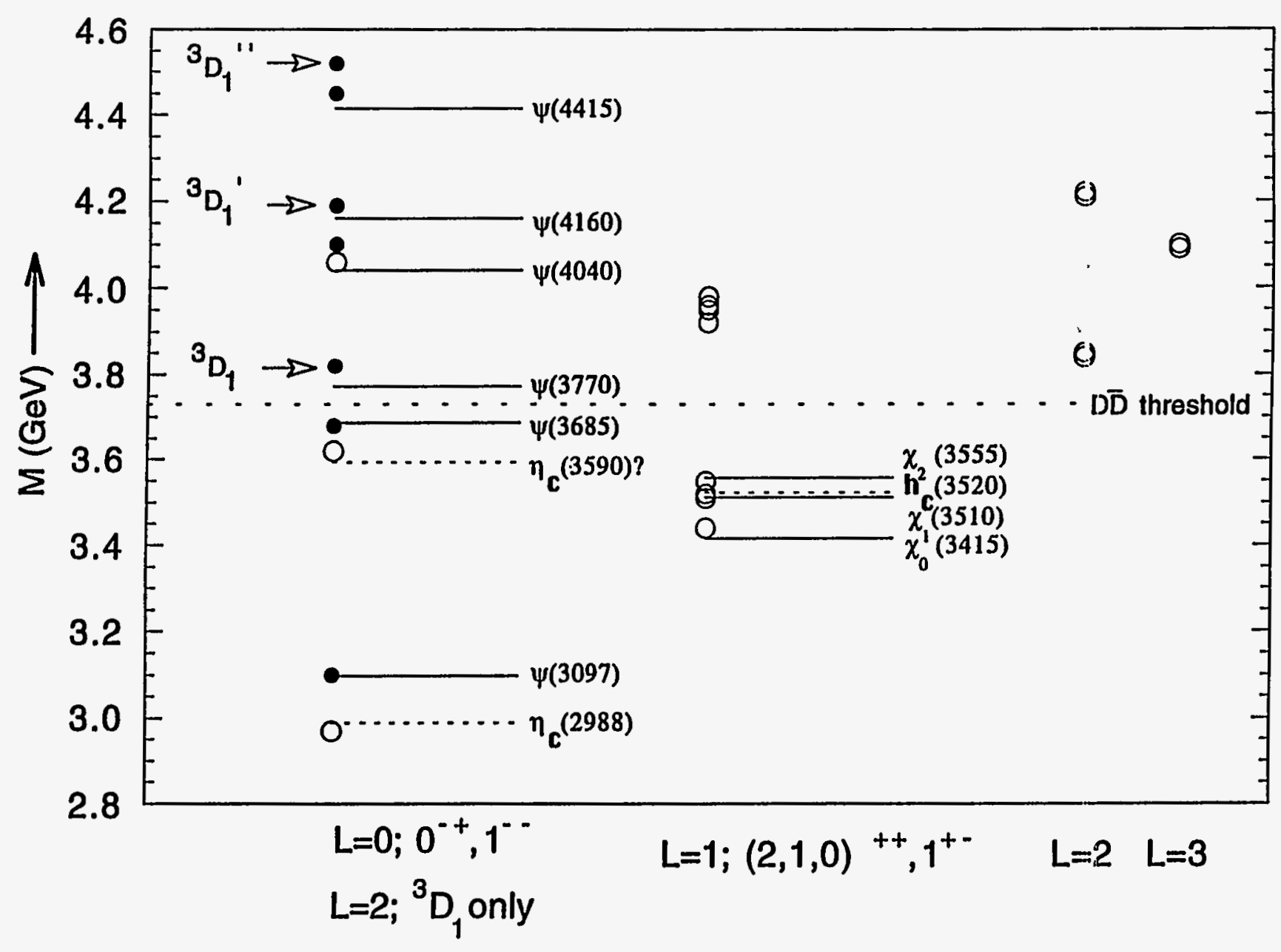




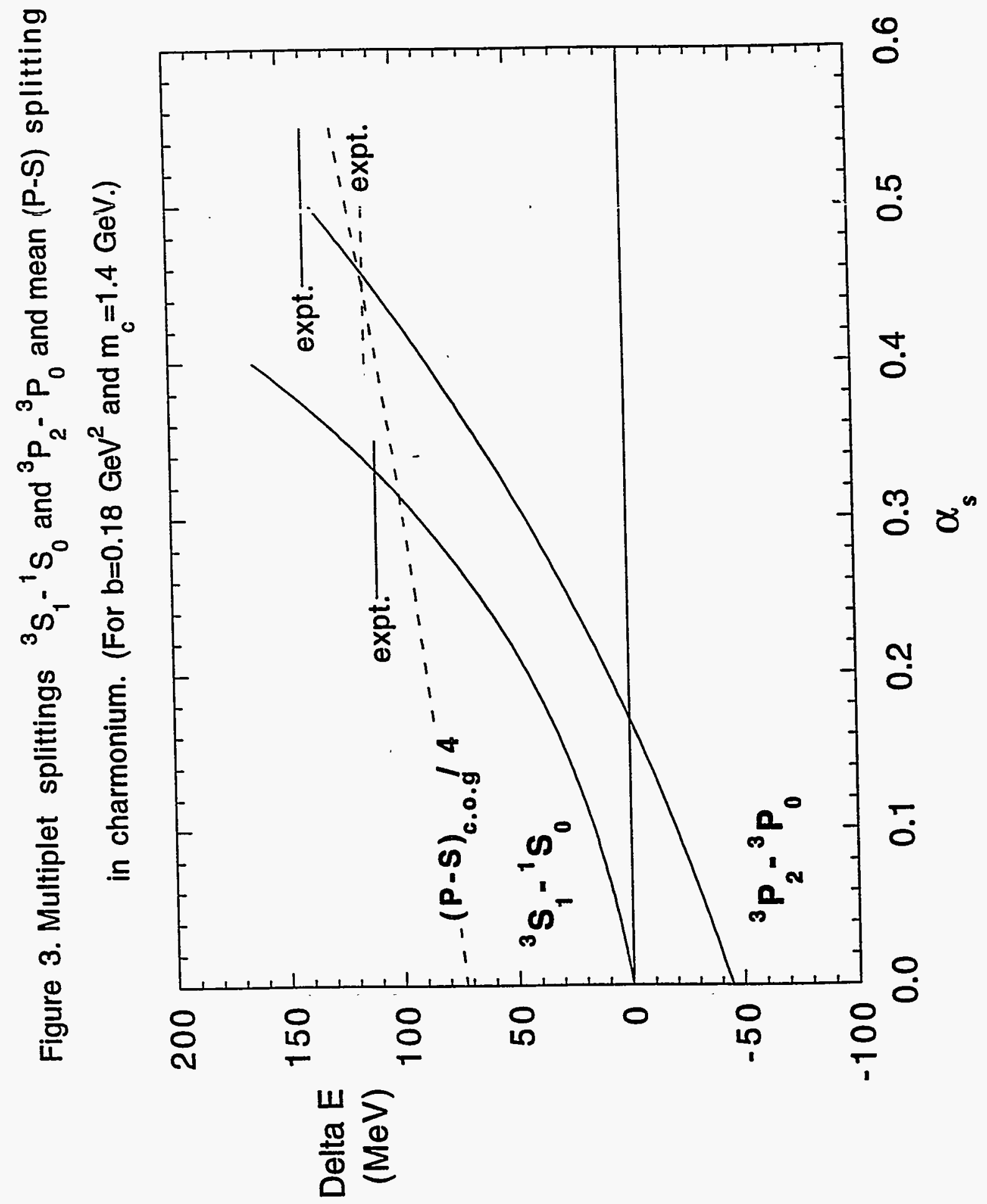




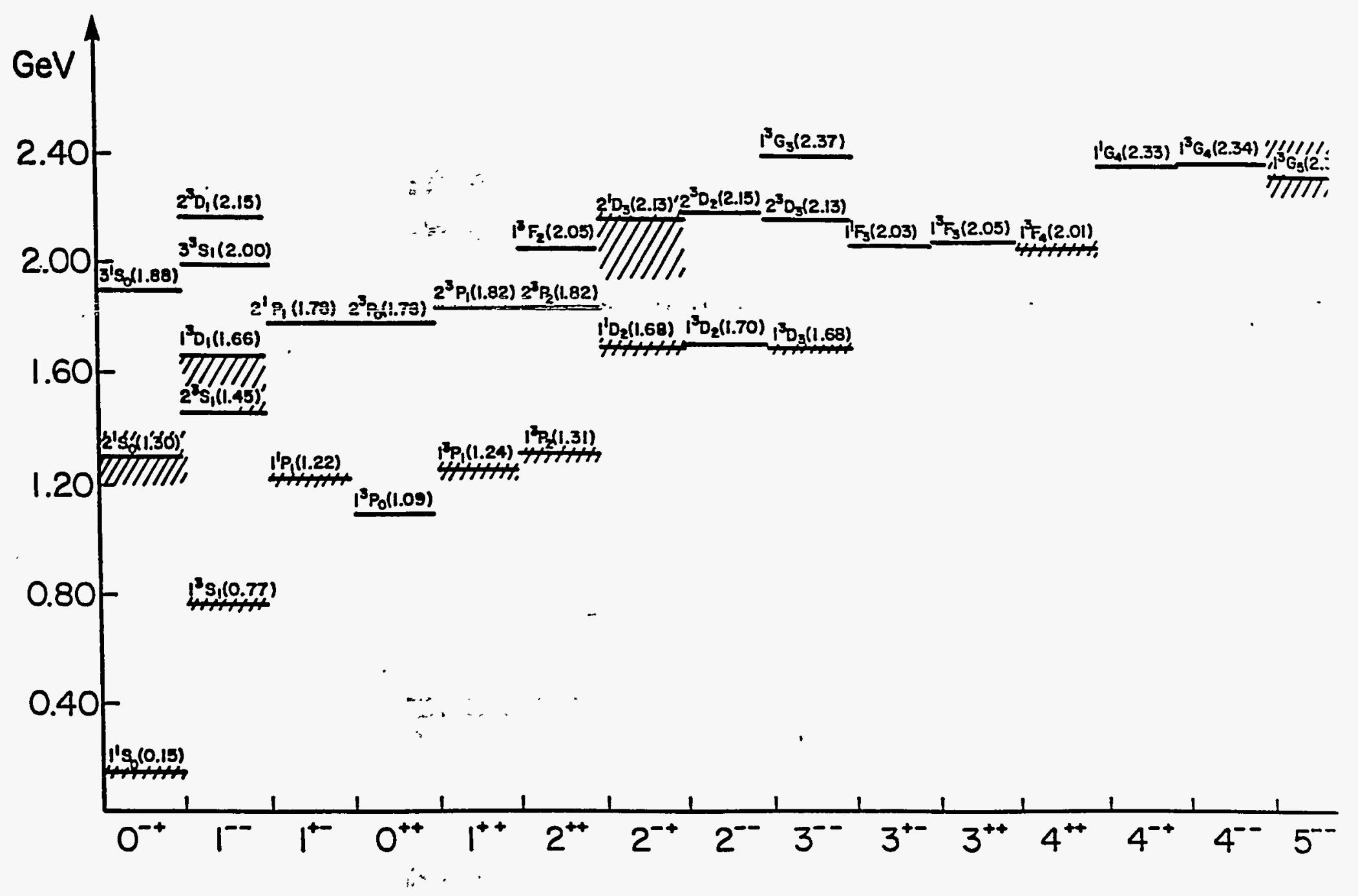

Figure 4. I=1 meson resonances, as of 1985; experimental (shaded) and theoretical qqbar levels (lines) (Godfrey and Isgur [3]). 


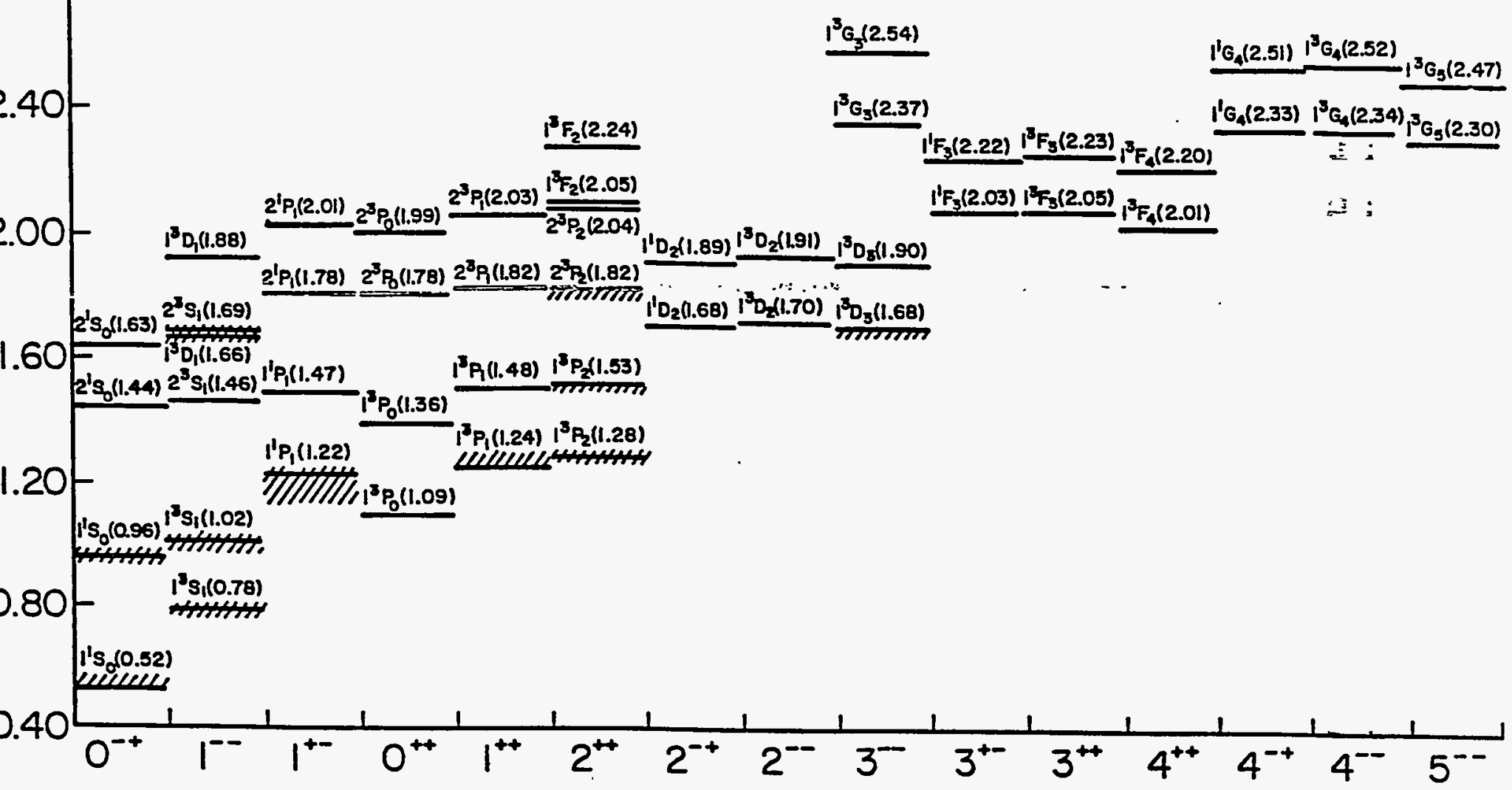

Figure 5. As in Figure 4 but for $l=0$ states; uubar, ddbar and ssbar. 